\title{
Analysis of Ridged Circular Waveguides by the Coupled-Integral-Equations Technique
}

\author{
Smain Amari, Séverine Catreux, Rüdiger Vahldieck, Senior Member, IEEE, \\ and Jens Bornemann, Senior Member, IEEE
}

\begin{abstract}
Cutoff wavenumbers of transverse electric (TE) and transverse magnetic (TM) modes of ridged circular waveguides are accurately determined using the coupled-integral-equations technique (CIET). A set of coupled integral equations for the electric field at the interfaces are derived and then solved by the moment method. Basis functions which include the edge conditions at all metallic edges are used. Results from this paper are compared with available data to demonstrate the accuracy and efficiency of the approach.
\end{abstract}

Index Terms - Circular waveguides, integral equations, ridge waveguides, waveguide theory.

\section{INTRODUCTION}

$\mathbf{R}$ IDGE waveguides are often encountered in microwave devices where broad-band operation is required, and in dual-mode microwave filters and polarizers. Accurate analysis and design of these devices is achievable only through an efficient account of the eigenvalues and eigenfields of the ridged section and the coupling which takes place at the discontinuities.

In dual-mode filters, a large number of modes in the ridged section are necessary to describe the scattering at the transition from hollow-to-ridged circular waveguide. It is, therefore, of prime importance to dispose of an efficient method to determine the cutoff wavenumbers and the corresponding coupling coefficients of an arbitrarily large number of modes for arbitrary dimensions and locations of the metallic ridge.

A variety of numerical techniques have been used to tackle this problem. The finite-element method (FEM) was used in the analysis and design of a compact polarizer [1]. Sun and Balanis applied the magnetic-field integral equation (MFIE) using pulses as basis functions in a moment-method solution for the cutoff frequencies of transverse electric (TE) and transverse magnetic (TM) modes of structures with two symmetry planes [2]. The mode-matching technique (MMT) was used by Balaji and Vahldieck to determine the onset of the fundamental and higher order modes in ridged circular waveguides [3]. The method of lines (MoL) was applied to the problem of symmetric ridged circular waveguide with partial dielectric

Manuscript received September 4, 1996; revised February 13, 1998.

S. Amari, S. Catreux, and J. Bornemann are with the Department of Electrical and Computer Engineering, Laboratory for Lightwave Electronics, Microwaves, and Communications (LLiMiC), University of Victoria, Victoria, B.C., V8W 3P6 Canada.

R. Vahldieck is with the Laboratory for Electromagnetic Fields and Microwave Electronics, Swiss Federal Institute of Technology ETH Zentrum, Gloriastrasse 35, CH-8092 Zürich, Switzerland.

Publisher Item Identifier S 0018-9480(98)03158-5. filling [4]. The generalized spectral-domain approach (GSDA) was applied to ridged circular waveguides with two or more symmetry planes [5].

The standard MMT exhibits the phenomenon of relative convergence in addition to slow numerical convergence when sharp metallic edges are present. On the other hand, the GSDA, which successfully includes the edge condition, relies for its efficiency on finding closed-form expressions for its infinite modal double sums [5]. The additional and arguably artificial radial sums appear in the GSDA because equivalent electric and magnetic current densities at interfaces are defined using short-circuited gaps [5].

The coupled-integral-equations technique (CIET), which is applied to the ridged circular waveguide in this paper, includes the edge condition, eliminates the phenomenon of relative convergence, and, from the outset, avoids the additional sums which appear in the GSDA. In this technique, a set of coupled integral equations in the tangential electric fields at the gaps of the interfaces are derived and solved by the moment method using basis functions which include the edge conditions. The phenomenon of relative convergence is overcome by emphasizing the fact that the dominant physics of the problem takes place at the sharp metallic edges of the ridges and reformulating the problem in terms of the true tangential electric fields at the different interfaces. The "modes" of the subregions into which the original structure is divided are given a minor role, primarily in computing inner products. The additional radial sums are eliminated by imposing only the physical boundary conditions to the "modes" of each of the subregions. Finally, the edge conditions, at eventually more than one ridge, are systematically included in the basis functions, thereby ensuring numerical efficiency and fast convergence. The CIET handles both symmetric and asymmetric structures, albeit the ridges are assumed to fit into the polar system of coordinates.

The CIET is first applied to determine the TE and TM spectra of a single-ridge circular waveguide and is described in ample details. To show its versatility and efficiency, a structure with two ridges of arbitrary thickness and locations is then analyzed.

\section{ANAYlsis of Single Ridge Circular WaVeguide}

The CIET is first applied to the structure shown in Fig. 1. It consists of a metallic ridge of inner radius $b$ and outer thickness $2 \theta$ in a lossless metallic circular waveguide of radius $a$. As the structure (which is assumed infinitely long in the $z$-direction) is air-filled, it is sufficient to determine the cutoff wavenumbers 


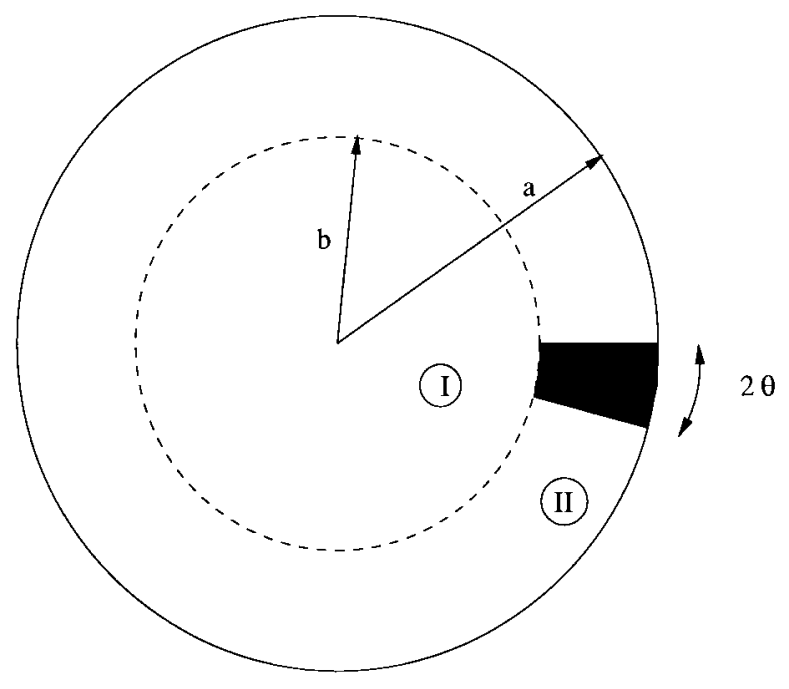

Fig. 1. Cross section of a ridged circular waveguide and its subregions for analysis.

in order to account for the propagation properties of the different modes at arbitrary frequencies.

\section{A. Cutoff Wavenumbers of TM Modes}

In order to determine the cutoff wavenumbers of TM modes, we subdivide the structure into the two regions shown in Fig. 1. In each region, the axial electric field $E_{z}$ is expanded in a series of "modes," each of which satisfies all the boundary conditions except at the interface between the two regions and over the metallic surface of the ridge at $\rho=b$. At cutoff, the only nonvanishing components of the electromagnetic field, which are tangential to the interface I-II, are $E_{z}$ and $H_{\phi}$. It is, therefore, sufficient to enforce the boundary conditions of these two quantities at the interface. Both polarizations (cos and $\sin$ ) are analyzed simultaneously.

In Region I, the axial component of the electric field $E_{z}^{I}$ is expanded in a series of the form

$$
E_{z}^{I}(\rho, \phi)=\sum_{n=0}^{\infty} J_{n}\left(k_{c} \rho\right)\left[A_{n} \cos (n \phi)+B_{n} \sin (n \phi)\right] .
$$

In Region II, the modal expansion is chosen to satisfy the boundary conditions at $\rho=a, \phi=0$, and $\phi=2(\pi-\theta)$ :

$$
\begin{aligned}
E_{z}^{I I}(\rho, \phi)= & \sum_{m=1}^{\infty} C_{m}\left[J_{l}\left(k_{c} a\right) Y_{l}\left(k_{c} \rho\right)\right. \\
& \left.-Y_{l}\left(k_{c} a\right) J_{l}\left(k_{c} \rho\right)\right] \sin \left[\frac{m \pi \phi}{2(\pi-\theta)}\right] .
\end{aligned}
$$

Here, $l=m \pi / 2(\pi-\theta), J_{l}$, and $Y_{l}$ are Bessel and Neumann functions of order $l$, respectively.

Note that in both regions, we do not impose any specific boundary conditions at the interface I-II, as this would quantize the wavenumbers in the radial direction and introduce an additional sum.

The boundary conditions at the interface are

$$
\begin{aligned}
& E_{z}^{I}(\rho=b, \phi)=0, \quad 2(\pi-\theta) \leq \phi \leq 2 \pi \\
& E_{z}^{I}(\rho=b, \phi)=E_{z}^{I I}(\rho=b, \phi), \quad 0 \leq \phi \leq 2(\pi-\theta)
\end{aligned}
$$

and

$$
H_{\phi}^{I}(\rho=b, \phi)=H_{\phi}^{I I}(\rho=b, \phi), \quad 0 \leq \phi \leq 2(\pi-\theta) .
$$

At this point, we depart from the standard MMT where the modal expansions in (1) and (2) are directly used in (3) and instead introduce an additional degree of freedom in the problem. Let us assume that the true tangential electric field $E_{z}$ at the interface I-II is given by an unknown function $X(\phi)$. It is then possible to combine (3a) and (3b) into a single equation which guarantees that each one of them is always satisfied. Indeed, if we require that the function $X(\phi)$ satisfies the following condition

$$
X(\phi)=0, \quad 2(\pi-\theta) \leq \phi \leq 2 \pi
$$

and rewrite $(3 b)$ in the form

$$
\begin{array}{r}
E_{z}^{I}(\rho=b, \phi)=X(\phi) \\
E_{z}^{I I}(\rho, \phi)=X(\phi)
\end{array}
$$

then the boundary conditions (3a) and (3b) are automatically satisfied.

To determine the function $X(\phi)$, the modal expansions in (1) and (2) are used in (5) to project out the modal coefficients $A_{n}, B_{n}$, and $C_{m}$ leading to

$$
\begin{aligned}
A_{n} & =\frac{1}{J_{n}\left(k_{c} b\right)} \frac{1}{\pi\left(1+\delta_{n 0}\right)} \\
\int_{0}^{2(\pi-\theta)} X(\phi) \cos (n \phi) d \phi & =\frac{\tilde{X}^{I c}(n)}{J_{n}\left(k_{c} b\right)} \\
B_{n} & =\frac{1}{J_{n}\left(k_{c} b\right)} \frac{1}{\pi} \\
\int_{0}^{2(\pi-\theta)} X(\phi) \sin (n \phi) d \phi & =\frac{\tilde{X}^{I c}(n)}{J_{n}\left(k_{c} b\right)}
\end{aligned}
$$

and

$$
\begin{aligned}
C_{m} & =\frac{1}{(\pi-\theta)} \frac{\int_{0}^{2(\pi-\theta)} X(\phi) \sin \left[\frac{m \pi \phi}{2(\pi-\theta)}\right] d \phi}{Y_{l}\left(k_{c} a\right) J_{l}\left(k_{c} b\right)-J_{l}\left(k_{c} a\right) Y_{l}\left(k_{c} b\right)} \\
& =\frac{\tilde{X}^{I I s}(m)}{Y_{l}\left(k_{c} a\right) J_{l}\left(k_{c} b\right)-J_{l}\left(k_{c} a\right) Y_{l}\left(k_{c} b\right)} .
\end{aligned}
$$

The following notations were introduced for convenience:

$$
\begin{aligned}
\tilde{X}^{I c}(n) & =\frac{1}{\pi\left(1+\delta_{n 0}\right)} \int_{0}^{2(\pi-\theta)} X(\phi) \cos (n \phi) d \phi \\
\tilde{X}^{I s}(n) & =\frac{1}{\pi} \int_{0}^{2(\pi-\theta)} X(\phi) \sin (n \phi) d \phi \\
\tilde{X}^{I I s}(m) & =\frac{1}{(\pi-\theta)} \int_{0}^{2(\pi-\theta)} X(\phi) \sin \left[\frac{m \pi \phi}{2(\pi-\theta)}\right] d \phi
\end{aligned}
$$

At this point, it only remains to enforce the continuity of the tangential magnetic field at the interface [i.e., (3c)]. Recall that in cylindrical coordinates $H_{\phi} \propto \partial E_{z} / \partial \rho$. If the modal expansion coefficients, as given by (6), are used in (1) and (2), 
which are in turn used in (3c), we obtain an integral equation for the function $X(\phi)$, namely

$$
\begin{aligned}
& \sum_{m=1}^{\infty} \frac{J_{l}\left(k_{c} a\right) Y_{l}^{\prime}\left(k_{c} b\right)-Y_{l}\left(k_{c} a\right) J_{l}^{\prime}\left(k_{c} b\right)}{J_{l}\left(k_{c} a\right) Y_{l}\left(k_{c} b\right)-Y_{l}\left(k_{c} a\right) J_{l}\left(k_{c} b\right)} \tilde{X}^{I I}(m) \sin \left[\frac{m \pi \phi}{2(\pi-\theta)}\right]-\sum_{n=0}^{\infty} \frac{J_{n}^{\prime}\left(k_{c} b\right)}{J_{n}\left(k_{c} b\right)} \\
& \cdot\left[\tilde{X}^{I c}(n) \cos (n \phi)+\tilde{X}^{I s}(n) \sin (n \phi)\right]=0 .
\end{aligned}
$$

Note that the integral equation (8) holds only along the interface I-II [i.e., when $0 \leq \phi \leq 2(\pi-\theta)$ ].

This integral equation is solved by the moment method [6]. To achieve numerical efficiency, we expand the function $X(\phi)$ in a series of basis functions which contain as much as possible of the a priori information we have on the behavior of the tangential electric field at the interface, especially its nonanalytic nature at the sharp metallic edges of the ridge. Let $B_{i}(\phi)$ denote a generic element of such a set of basis functions such that

$$
X(\phi)=\sum_{i=1}^{M} c_{i} B_{i}(\phi) .
$$

The number of terms in this expansion, the value of $M$, is increased until convergence is achieved. It will be seen, however, that only a few terms are needed to accurately determine the spectrum of the structure when basis functions which contain the edge conditions are used.

To determine the constants $c_{i}$, we apply Galerkin's method to the integral equation (8) with (9). A homogeneous linear set of equations in the coefficients $c_{i}$ results, namely

$$
[A][c]=0 .
$$

The entries of the square and symmetric matrix $[A]$ are given by

$$
\begin{aligned}
{[A]_{i j}=} & \sum_{n=0}^{\infty} \frac{J_{n}^{\prime}\left(k_{c} b\right)}{J_{n}\left(k_{c} b\right)} \\
& \cdot\left[\tilde{B}_{i}^{I c}(n) \tilde{B}_{j}^{I c}(n)\left(1+\delta_{n 0}\right)+\tilde{B}_{i}^{I s}(n) \tilde{B}_{j}^{I s}(n)\right] \\
& -\left(1-\frac{\theta}{\pi}\right) \sum_{m=1}^{\infty} \frac{J_{l}\left(k_{c} a\right) Y_{l}^{\prime}\left(k_{c} b\right)-Y_{l}\left(k_{c} a\right) J_{l}^{\prime}\left(k_{c} b\right)}{J_{l}\left(k_{c} a\right) Y_{l}\left(k_{c} b\right)-J_{l}\left(k_{c} a\right) Y_{l}\left(k_{c} b\right)} \\
& \cdot \tilde{B}_{i}^{I I}(m) \tilde{B}_{j}^{I I}(m) .
\end{aligned}
$$

The cutoff wavenumbers $k_{c}$ are determined as the roots of the determinant of the matrix $[A]$ or, to avoid the poles which are usually present in the determinant and, equivalently, as the zero of its smallest singular value [7]. It is worth emphasizing the way the modes of the two subregions appear in the theory; they are used only in computing the inner products which appear in the matrix elements $[A]_{i j}$. These sums are tested for convergence, leaving only one parameter in the problem, the number of basis functions $M$, thereby eliminating the phenomenon of relative convergence.

\section{B. Basic Functions for TM Modes}

In order to guarantee numerical efficiency, the basis functions should include the nonanalytic nature of the axial component $E_{z}$ at the sharp metallic edges of the ridge. Since the internal angle of the metallic edge is equal to $\pi / 2$, the basis functions should vanish as $r^{2 / 3}$ as the radial distance from the edge $r$ approaches zero [8]. A set of basis functions which satisfy this local requirement is given by

$$
B_{k}(\phi)=\frac{\sin \left[\frac{k \pi \phi}{2(\pi-\theta)}\right]}{\{\phi[2(\pi-\theta)-\phi]\}^{1 / 3}}, \quad k=1,2,3, \cdots
$$

Note that these basis functions are perturbed versions of the angular field distributions of the modes of an empty circular waveguide. It is, therefore, expected that the cutoff wavenumber of a mode which corresponds to a root of $J_{n}\left(k_{c} a\right)$ is determined accurately only when the basis function of order $n$ is used. On the other hand, the remaining solutions corresponding to the higher order roots of $J_{n}\left(k_{c} a\right)$ are then satisfactorily determined from the same number of basis function as the numerical results illustrate.

The spectra of these functions [i.e., the integrals in (7)] can be expressed in terms of Bessel functions of the first kind of order 1/6 [9], as shown in (13a)-(13c), at the bottom of the following page.

When the argument of the Bessel function vanishes, which occurs when $m=k$ in (13c), the corresponding term should be replaced by

$$
\tilde{B}_{k}^{I I s}(k)=\frac{1}{2} \Gamma\left(\frac{1}{2}\right) \Gamma\left(\frac{2}{3}\right)\left[\frac{1}{\Gamma\left(\frac{7}{6}\right)}-\frac{\cos (k \pi) J_{1 / 6}(k \pi)}{\left(\frac{k \pi}{2}\right)^{1 / 6}}\right] .
$$

\section{Cutoff Wavenumbers of TE Modes}

The cutoff wavenumbers of the TE modes can be determined following an analogous analysis to that of the TM modes.

The axial component of the magnetic field, from which the transverse components of the electromagnetic fields are obtained, is expanded in modal series in each of the two regions. The boundary conditions at the metallic surfaces of Region II are included in these expansions while no specific conditions are imposed at the interface I-II:

$$
H_{z}^{I}(\rho, \phi)=\sum_{n=0}^{\infty} J_{n}\left(k_{c} \rho\right)\left[D_{n} \cos (n \phi)+E_{n} \sin (n \phi)\right]
$$

and

$$
\begin{aligned}
H_{z}^{I I}(\rho, \phi)= & \sum_{m=0}^{\infty} F_{m}\left[J_{l}^{\prime}\left(k_{c} a\right) Y_{l}\left(k_{c} \rho\right)\right. \\
& \left.-Y_{l}^{\prime}\left(k_{c} a\right) J_{l}\left(k_{c} \rho\right)\right] \cos \left[\frac{m \pi \phi}{2(\pi-\theta)}\right] .
\end{aligned}
$$

The boundary conditions of the TE modes at cutoff correspond to the vanishing of $E_{\phi}$ over the metallic surface of the ridge 
and the continuity of $E_{\phi}$ and $H_{z}$ along the interface I-II, i.e.,

$$
\begin{aligned}
& E_{\phi}^{I}(\rho=b, \phi)=0, \quad 2(\pi-\theta) \leq \phi \leq 2 \pi \\
& E_{\phi}^{I}(\rho=b, \phi)=E_{\phi}^{I I}(\rho=b, \phi), \quad 0 \leq \phi \leq 2(\pi-\theta)
\end{aligned}
$$

and

$$
H_{z}^{I}(\rho=b, \phi)=H_{z}^{I I}(\rho=b, \phi), \quad 0 \leq \phi \leq 2(\pi-\theta) .
$$

To enforce the boundary conditions of the electric field [(16a) and (16b)], we assume that the tangential electric field $E_{\phi}$ at the interface is given by an unknown function $Y(\phi)$ which vanishes over the metallic part of the interface I-II:

$$
Y(\phi)=0, \quad 2(\pi-\theta) \leq \phi \leq 2 \pi
$$

and rewrite (16b) in the form

$$
\begin{aligned}
E_{\phi}^{I}(\rho=b, \phi) & =Y(\phi) \\
E_{\phi}^{I I}(\rho=b, \phi) & =Y(\phi) .
\end{aligned}
$$

Using the expansions of $H_{z}$ in Regions I and II in (18), we can express the modal expansion coefficients, $D_{n}, E_{n}$, and $F_{m}$ in terms of the spectra of $Y(\phi)$, which are then used in the continuity of the magnetic field $H_{z}$ to derive the following integral equation for $Y(\phi)$ :

$$
\begin{gathered}
\sum_{m=0}^{\infty} \tilde{Y}^{I I c}(m) \frac{Y_{l}^{\prime}\left(k_{c} a\right) J_{l}\left(k_{c} b\right)-J_{l}^{\prime}\left(k_{c} a\right) Y_{l}\left(k_{c} b\right)}{Y_{l}^{\prime}\left(k_{c} a\right) J_{l}^{\prime}\left(k_{c} b\right)-J_{l}^{\prime}\left(k_{c} a\right) Y_{l}^{\prime}\left(k_{c} b\right)} \\
\cdot \cos \left[\frac{m \pi \phi}{2(\pi-\theta)}\right]-\sum_{n=0}^{\infty} \frac{J_{n}\left(k_{c} b\right)}{J_{n}^{\prime}\left(k_{c} b\right)} \\
\cdot\left[\tilde{Y}^{I c}(n) \cos (n \phi)+\tilde{Y}^{I s}(n) \sin (n \phi)\right]=0 .
\end{gathered}
$$

Again, this equation holds only when $\phi \in[0,2(\pi-\theta)]$.
To solve this integral equation, we expand the function $Y(\phi)$ in a series of basis functions which satisfy condition (18) along with the edge conditions. Let $B_{i}$ denote a generic element of such a set of basis functions

$$
Y(\phi)=\sum_{i=1}^{M} d_{i} B_{i}(\phi) .
$$

Applying Galerkin's method, we obtain a set of linear equations in the coefficients $d_{i}$ :

$$
[G][d]=0
$$

where the entries of the matrix $[G]$ are given by

$$
\begin{aligned}
{[G]_{i j}=} & \left(\frac{1-\theta}{\pi}\right) \sum_{m=0}^{\infty}\left(1+\delta_{m 0}\right) \tilde{B}_{i}^{I I c}(m) \tilde{B}_{j}^{I I c}(m) \\
& \cdot \frac{Y_{l}^{\prime}\left(k_{c} a\right) J_{l}\left(k_{c} b\right)-J_{l}^{\prime}\left(k_{c} a\right) Y_{l}\left(k_{c} b\right)}{Y_{l}^{\prime}\left(k_{c} a\right) J_{l}^{\prime}\left(k_{c} b\right)-J_{l}^{\prime}\left(k_{c} a\right) Y_{l}^{\prime}\left(k_{c} b\right)}-\sum_{n=0}^{\infty} \frac{J_{n}\left(k_{c} b\right)}{J_{n}^{\prime}\left(k_{c} b\right)} \\
& \cdot\left[\tilde{B}_{i}^{I c}(n) \tilde{B}_{j}^{I c}(n)\left(1+\delta_{n 0}\right)+\tilde{B}_{i}^{I s}(n) \tilde{B}_{j}^{I s}(n)\right] .
\end{aligned}
$$

As in the TM case, the cutoff wavenumbers are determined as the roots of the determinant of the square and symmetric matrix $[G]$. To avoid the poles in the determinant, the zeros of the smallest singular value of this matrix are located instead [7].

\section{Basis Functions for TE Modes}

The basis functions used to expand $E_{\phi}$ at the interface should capture the salient features of this quantity to guarantee numerical efficiency. Since $E_{\phi}$ is normal to the axis of the $90^{\circ}$ metallic wedge, it becomes singular as $r^{-1 / 3}$ as the radial

$$
\begin{aligned}
& \tilde{B}_{k}^{I c}(n)=\frac{1}{2\left(1+\delta_{n 0}\right)}\left(1-\frac{\theta}{\pi}\right) \Gamma(1 / 2) \Gamma(2 / 3)\left\{\frac{J_{1 / 6}\left[k \frac{\pi}{2}+n(\pi-\theta)\right]}{\left|k \frac{\pi}{4}+n \frac{(\pi-\theta)}{2}\right|^{1 / 6}} \sin \left[k \frac{\pi}{2}+n(\pi-\theta)\right]\right. \\
& \left.+\frac{J_{1 / 6}\left[\left|k \frac{\pi}{2}-n(\pi-\theta)\right|\right]}{\left|k \frac{\pi}{4}-n \frac{(\pi-\theta)}{2}\right|^{1 / 6}} \sin \left[k \frac{\pi}{2}-n(\pi-\theta)\right]\right\} \\
& \tilde{B}_{k}^{I s}(n)=\frac{1}{2}\left(1-\frac{\theta}{\pi}\right) \Gamma(1 / 2) \Gamma(2 / 3)\left\{-\frac{J_{1 / 6}\left[k \frac{\pi}{2}+n(\pi-\theta)\right]}{\left|k \frac{\pi}{4}+n \frac{(\pi-\theta)}{2}\right|^{1 / 6}} \cos \left[k \frac{\pi}{2}+n(\pi-\theta)\right]\right. \\
& \left.+\frac{J_{1 / 6}\left[\left|k \frac{\pi}{2}-n(\pi-\theta)\right|\right]}{\left|k \frac{\pi}{4}-n \frac{(\pi-\theta)}{2}\right|^{1 / 6}} \cos \left[k \frac{\pi}{2}-n(\pi-\theta)\right]\right\} \\
& \tilde{B}_{k}^{I I s}(m)=\frac{1}{2} \Gamma(1 / 2) \Gamma(2 / 3)\left\{\frac{J_{1 / 6}\left[|m-k| \frac{\pi}{2}\right]}{\left|(m-k) \frac{\pi}{4}\right|^{1 / 6}} \cos \left[(m-k) \frac{\pi}{2}\right]-\frac{J_{1 / 6}\left[(m+k) \frac{\pi}{2}\right]}{\left|(m+k) \frac{\pi}{4}\right|^{1 / 6}} \cos \left[(m+k) \frac{\pi}{2}\right]\right\}
\end{aligned}
$$


distance to the axis of the wedge $r$ approaches zero [8]. Taking into account the fact that there are two wedges located at $\phi=0$ and $\phi=2(\pi-\theta)$, a set of basis functions which satisfy these requirements is given by

$$
B_{k}(\phi)=\frac{\cos \left[\frac{(k-1) \pi \phi}{2(\pi-\theta)}\right]}{\{\phi[2(\pi-\theta)-\phi]\}^{1 / 3}}, \quad k=1,2, \cdots .
$$

The spectra of these basis functions in each of the two regions can be expressed in terms of Bessel function of the first kind of order 1/6 [9], as shown in (24a)-(24c), at the bottom of the page.

Note that the modes of the two subregions are again all included in the theory, and in a rather simple way; they appear only in computing the inner products in the quantities $[G]_{i j}$. The phenomenon of relative convergence is eliminated by testing these sums for convergence, thereby leaving only one free parameter in the numerical solution, i.e., the number of basis functions $M$.

This analysis shows how an integral equation for an illbehaved quantity (the tangential electric field at the interface) can be derived and solved. The case where more than one ridge is present in the structure and where no symmetry is present requires a set of coupled integral equations instead. The following sections present an analysis of a double-ridge structure which is eventually intended for dual-mode filters.

\section{ANALysis of Asymmetric Double-Ridge WaVeguide}

The structure under consideration is shown in Fig. 2. The metallic walls are assumed lossless, the locations and thicknesses of the two ridges arbitrary. For simplicity, the analysis

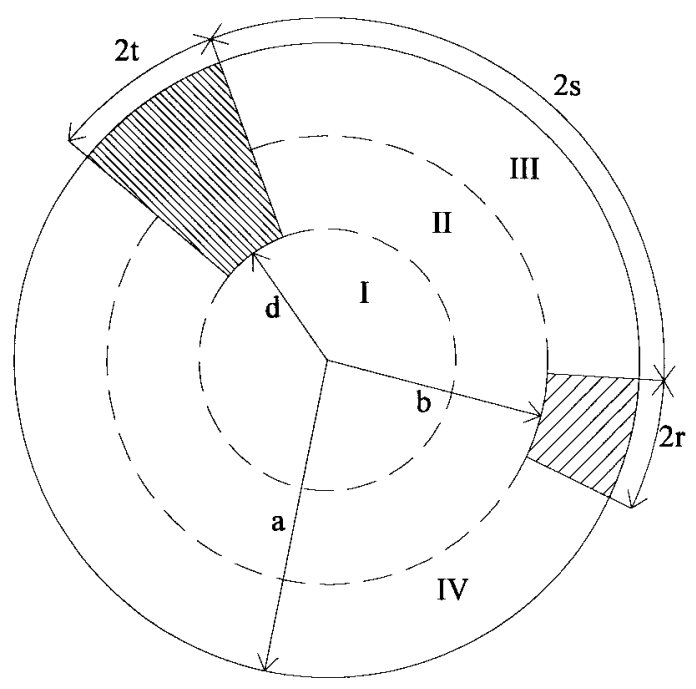

Fig. 2. Cross section of asymmetric double-ridge circular waveguide and its subregions for analysis.

is limited to the case where $d<b$ and the depth of the two ridges are smaller than the radius $a$ of the empty cylinder. The analysis is straightforwardly adapted to other situations such as two ridges of equal depths, or one ridge exceeding the center of the empty cylinder. We first consider the cutoff wavenumbers of the TM modes.

\section{A CutofF WAVEnumbers of TM MOdes}

The cross section of the structure is divided into four subregions, as shown in Fig. 2. In each of these regions, the potential of the TM modes are expanded in modal series which include as much of the boundary conditions as possible.

$$
\begin{aligned}
& \tilde{B}_{k}^{I c}(n)=\frac{1}{2\left(1+\delta_{n 0}\right)}\left(1-\frac{\theta}{\pi}\right) \Gamma\left(\frac{1}{2}\right) \Gamma\left(\frac{2}{3}\right) \frac{J_{1 / 6}\left[(k-1) \frac{\pi}{2}+n(\pi-\theta)\right]}{\left|(k-1) \frac{\pi}{4}+n \frac{(\pi-\theta)}{2}\right|^{1 / 6}} \\
& \times \cos \left[(k-1) \frac{\pi}{2}+n(\pi-\theta)\right]+\frac{J_{1 / 6}\left[\left|(k-1) \frac{\pi}{2}-n(\pi-\theta)\right|\right]}{\left|(k-1) \frac{\pi}{4}-n \frac{(\pi-\theta)}{2}\right|^{1 / 6}} \cos \left[(k-1) \frac{\pi}{2}-n(\pi-\theta)\right] \\
& \tilde{B}_{k}^{I s}(n)=\frac{1}{2}\left(1-\frac{\theta}{\pi}\right) \Gamma\left(\frac{1}{2}\right) \Gamma\left(\frac{2}{3}\right) \frac{J_{1 / 6}\left[(k-1) \frac{\pi}{2}+n(\pi-\theta)\right]}{\left|(k-1) \frac{\pi}{4}+n \frac{(\pi-\theta)}{2}\right|^{1 / 6}}
\end{aligned}
$$

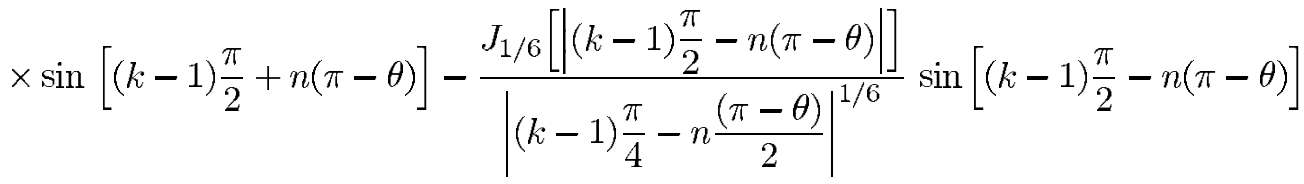

$$
\begin{aligned}
& \tilde{B}_{k}^{I I c}(m)=\frac{\Gamma\left(\frac{1}{2}\right) \Gamma\left(\frac{2}{3}\right)}{\left(1+\delta_{m 0}\right)} \frac{J_{1 / 6}\left\{|m-(k-1)| \frac{\pi}{2}\right\}}{\left|[m-(k-1)] \frac{\pi}{4}\right|^{1 / 6}} \cos \left\{[m-(k-1)] \frac{\pi}{2}\right\}+\frac{J_{1 / 6}\left\{[m+(k-1)] \frac{\pi}{2}\right\}}{\left|[m+(k-1)] \frac{\pi}{4}\right|^{1 / 6}} \cos \left\{[m+(k-1)] \frac{\pi}{2}\right\}
\end{aligned}
$$


However, we do not impose any conditions at the interfaces between the different adjacent subregions. Taking into account the fact that $E_{z}$ is proportional to the potential, we have modal expansions (25a)-(25d), shown at the bottom of the page. Here, $l=[m \pi / 2(\pi-t)], \mu=(m \pi / 2 s)$, and $\eta=$ $[m \pi / 2(\pi-r-s-t)]$.

At cutoff, the only nonvanishing components of the electromagnetic field which are tangential to the interfaces are $E_{z}$ and $H_{\phi}$, which is proportional to $\partial E_{z} / \partial \rho$.

The boundary conditions of the TM modes are the continuity of the tangential electric and magnetic fields at the interfaces and the vanishing of $E_{z}$ over the metallic portions of these interfaces, namely

$$
\begin{gathered}
E_{z}^{I}(\rho=d, \phi)=0, \quad 2 s \leq \phi \leq 2(s+t) \\
E_{z}^{I I}(\rho=b, \phi)=0, \quad 2(\pi-r-s-t) \leq \phi \leq 2 \pi \\
E_{z}^{I}(\rho=d, \phi)=E_{z}^{I I}(\rho=d, \phi), \\
\quad-2(\pi-s-t) \leq \phi \leq 2 s, \quad(26 \mathrm{c}) \\
E_{z}^{I I}(\rho=b, \phi)=E_{z}^{I I I}(\rho=b, \phi), \quad 0 \leq \phi \leq 2 s \\
E_{z}^{I I}(\rho=b, \phi)=E_{z}^{I V}(\rho=b, \phi), \\
\quad-2(\pi-s-t) \leq \phi \leq-2 r
\end{gathered}
$$

$$
\begin{gathered}
H_{\phi}^{I}(\rho=d, \phi)=H_{\phi}^{I I}(\rho=d, \phi), \\
\quad-2(\pi-s-t) \leq \phi \leq 2 s \\
H_{\phi}^{I I}(\rho=b, \phi)=H_{\phi}^{I I I}(\rho=b, \phi), \quad 0 \leq \phi \leq 2 s
\end{gathered}
$$

and

$$
\begin{aligned}
H_{\phi}^{I I}(\rho=b, \phi)=H_{\phi}^{I V} & (\rho=b, \phi), \\
& -2(\pi-s-t) \leq \phi \leq-2 r .
\end{aligned}
$$

To enforce the boundary conditions of the tangential electric field at the different interfaces, we introduce three unknown functions $X_{1}(\phi), X_{2}(\phi)$, and $X_{3}(\phi)$, which are equal to $E_{z}$ at the three interfaces I-II, II-III, and II-IV, respectively. We also require that $X_{1}(\phi), X_{2}(\phi)$, and $X_{3}(\phi)$ satisfy the following conditions:

$$
\begin{array}{ll}
X_{1}(\phi)=0, & 2 s \leq \phi \leq 2(s+t) \\
X_{2}(\phi)=0, & \text { unless } 0 \leq \phi \leq 2 s
\end{array}
$$

and

$$
X_{3}(\phi)=0, \quad \text { unless }-2(\pi-s-t) \leq \phi \leq-2 r .
$$

We now rewrite the boundary conditions (26c)-(26e) in the following form:

$$
\begin{aligned}
E_{z}^{I}(\rho=d, \phi) & =X_{1}(\phi) \\
E_{z}^{I I}(\rho=d, \phi) & =X_{1}(\phi) \\
E_{z}^{I I}(\rho=b, \phi) & =X_{2}(\phi) \\
E_{z}^{I I I}(\rho=b, \phi) & =X_{2}(\phi)
\end{aligned}
$$

and

$$
\begin{gathered}
E_{z}^{I I}(\rho=b, \phi)=X_{3}(\phi) \\
E_{z}^{I V}(\rho=b, \phi)=X_{3}(\phi) .
\end{gathered}
$$

It can be easily verified that the boundary conditions of the tangential electric field are now all satisfied as long as the functions $X_{1}(\phi), X_{2}(\phi)$, and $X_{3}(\phi)$ satisfy condition (27). To derive a set of coupled integral equations for these functions, the modal expansion coefficients in (25) are expressed in terms of the spectra of these functions in the four subregions and then substituted in the continuity conditions of the tangential magnetic field $H_{\phi}$. The algebra is straightforward and leads to the three coupled integral equations shown in (29), at the bottom of the following page, which holds when $\phi \in$ $[-2(\pi-s-t), 2 s]$, shown in (30), at the bottom of the following page, which holds when $\phi \in[0,2 s]$, and shown in (31), at the bottom of the following page, which holds when $\phi \in[-2(\pi-s-t),-2 r]$.

In these integral equations, the transformed functions $\tilde{X}$ are defined by (32a)-(32g), shown at the bottom of the following page.

To solve these three coupled integral equations, we expand the functions $X_{i}(\phi)$ into a series of appropriate basis functions. Let $Q_{i}(\phi), R_{i}(\phi)$, and $S_{i}(\phi)$ denote generic elements of the set of basis functions for $X_{1}(\phi), X_{2}(\phi)$, and $X_{3}(\phi)$, respectively, such that

$$
\begin{aligned}
& X_{1}(\phi)=\sum_{k=1}^{M} c_{k} Q_{k}(\phi) \\
& X_{2}(\phi)=\sum_{k=1}^{M} d_{k} R_{k}(\phi)
\end{aligned}
$$

and

$$
X_{3}(\phi)=\sum_{k=1}^{M} e_{k} S_{k}(\phi)
$$

$$
\begin{aligned}
E_{z}^{I}(\rho, \phi) & =\sum_{n=0}^{\infty} J_{n}\left(k_{c} \rho\right)\left[A_{n} \cos (n \phi)+B_{n} \sin (n \phi)\right] \\
E_{z}^{I I}(\rho, \phi) & =\sum_{m=1}^{\infty}\left[C_{m} J_{l}\left(k_{c} \rho\right)+D_{m} Y_{l}\left(k_{c} \rho\right)\right] \sin \left[\frac{m \pi(\phi-2 s)}{2(\pi-t)}\right] \\
E_{z}^{I I I}(\rho, \phi) & =\sum_{m=1}^{\infty} D_{m}\left[Y_{\mu}\left(k_{c} a\right) J_{\mu}\left(k_{c} \rho\right)-Y_{\mu}\left(k_{c} \rho\right) J_{\mu}\left(k_{c} a\right)\right] \sin \left[\frac{m \pi}{2 s} \phi\right] \\
E_{z}^{I V}(\rho, \phi) & =\sum_{m=1}^{\infty} E_{m}\left[Y_{\eta}\left(k_{c} a\right) J_{\eta}\left(k_{c} \rho\right)-Y_{\eta}\left(k_{c} \rho\right) J_{\eta}\left(k_{c} a\right)\right] \sin \left[\frac{m \pi(\phi+2 r)}{2(\pi-r-s-t)}\right]
\end{aligned}
$$


For simplicity, we take the same number of basis functions at each interface.

Following the standard moment method, we apply Galerkin's method to the integral equations with the expansions (33) resulting in a linear set of homogeneous equations in the expansion coefficients $c_{i}, d_{i}$, and $e_{i}$ as follows:

$$
\begin{aligned}
{[A][c]+[B][d]+[C][e] } & =0 \\
{[D][c]+[E][d]+[F][e] } & =0 \\
{[G][c]+[H][d]+[I][e] } & =0 .
\end{aligned}
$$

The entries of the matrices appearing in (34) are given in Appendix A.
The cutoff wavenumbers of the TM modes of the structure are obtained as the zeros of the determinant of the extended matrix appearing in (34) or, equivalently, the zeros of its smallest singular value.

\section{B. Basis Functions for TM Modes}

The following set of basis functions which include the edge conditions at each of the metallic wedges of the two ridges are used in this paper:

$$
\begin{aligned}
& Q_{k}(\phi)=\frac{\sin \left[\frac{k \pi(\pi-2 s)}{2(\pi-t)}\right]}{\left[(2 s-\phi)(\phi+2(\pi-s-t)]^{1 / 3}\right.}, \\
& k=1,2, \cdots
\end{aligned}
$$

$$
\begin{aligned}
& \sum_{m=1}^{\infty}\left\{\frac{\left[\tilde{X}_{2}^{I I s}(m)+\tilde{X}_{3}^{I I s}(m)\right]\left[Y_{l}^{\prime}\left(k_{c} d\right) J_{l}\left(k_{c} d\right)-J_{l}^{\prime}\left(k_{c} d\right) Y_{l}\left(k_{c} d\right)\right]}{J_{l}\left(k_{c} d\right) Y_{l}\left(k_{c} b\right)-J_{l}\left(k_{c} b\right) Y_{l}\left(k_{c} d\right)}+\frac{\tilde{X}_{1}^{I I s}(m)\left[J_{l}^{\prime}\left(k_{c} d\right) Y_{l}\left(k_{c} b\right)-Y_{l}^{\prime}\left(k_{c} d\right) J_{l}\left(k_{c} b\right)\right]}{J_{l}\left(k_{c} d\right) Y_{l}\left(k_{c} b\right)-J_{l}\left(k_{c} b\right) Y_{l}\left(k_{c} d\right)}\right\} \\
& \cdot \sin \left[\frac{m \pi(\phi-2 s)}{2(\pi-t)}\right]-\sum_{n=0}^{\infty} \frac{J_{n}^{\prime}\left(k_{c} d\right)}{J_{n}\left(k_{c} d\right)}\left[\tilde{X}_{1}^{I c}(n) \cos (n \phi)+\tilde{X}_{1}^{I s}(n) \sin (n \phi)\right]=0
\end{aligned}
$$

$$
\begin{aligned}
& \sum_{m=1}^{\infty}\left\{\left[\tilde{X}_{2}^{I I s}(m)+\tilde{X}_{3}^{I I s}(m)\right] \frac{\left.Y_{l}^{\prime}\left(k_{c} b\right) J_{l}\left(k_{c} d\right)-J_{l}^{\prime}\left(k_{c} b\right) Y_{l}\left(k_{c} d\right)\right]}{J_{l}\left(k_{c} d\right) Y_{l}\left(k_{c} b\right)-J_{l}\left(k_{c} b\right) Y_{l}\left(k_{c} d\right)}+\tilde{X}_{1}^{I I s}(m) \frac{\left.J_{l}^{\prime}\left(k_{c} b\right) Y_{l}\left(k_{c} b\right)-Y_{l}^{\prime}\left(k_{c} b\right) J_{l}\left(k_{c} b\right)\right]}{J_{l}\left(k_{c} d\right) Y_{l}\left(k_{c} b\right)-J_{l}\left(k_{c} b\right) Y_{l}\left(k_{c} d\right)}\right\} \\
& \cdot \sin \left[\frac{m \pi(\phi-2 s)}{2(\pi-t)}\right]-\sum_{m=1}^{\infty} \tilde{X}_{2}^{I I I s}(m) \frac{J_{\mu}^{\prime}\left(k_{c} b\right) Y_{\mu}\left(k_{c} a\right)-J_{\mu}\left(k_{c} a\right) Y_{\mu}^{\prime}\left(k_{c} b\right)}{J_{\mu}\left(k_{c} b\right) Y_{\mu}\left(k_{c} a\right)-J_{\mu}\left(k_{c} a\right) Y_{\mu}\left(k_{c} b\right)} \sin \left[\frac{m \pi \phi}{2 s}\right]=0
\end{aligned}
$$

$$
\begin{gathered}
\sum_{m=1}^{\infty}\left\{\left[\tilde{X}_{2}^{I I s}(m)+\tilde{X}_{3}^{I s}(m)\right] \frac{\left.Y_{l}^{\prime}\left(k_{c} b\right) J_{l}\left(k_{c} d\right)-J_{l}^{\prime}\left(k_{c} b\right) Y_{l}\left(k_{c} d\right)\right]}{J_{l}\left(k_{c} d\right) Y_{l}\left(k_{c} b\right)-J_{l}\left(k_{c} b\right) Y_{l}\left(k_{c} d\right)}+\tilde{X}_{1}^{I I s}(m) \frac{\left.J_{l}^{\prime}\left(k_{c} b\right) Y_{l}\left(k_{c} b\right)-Y_{l}^{\prime}\left(k_{c} b\right) J_{l}\left(k_{c} b\right)\right]}{J_{l}\left(k_{c} d\right) Y_{l}\left(k_{c} b\right)-J_{l}\left(k_{c} b\right) Y_{l}\left(k_{c} d\right)}\right\} \\
\cdot \sin \left[\frac{m \pi(\phi-2 s)}{2(\pi-t)}\right]-\sum_{m=1}^{\infty} \tilde{X}_{3}^{I V s}(m) \frac{J_{\eta}^{\prime}\left(k_{c} b\right) Y_{\eta}\left(k_{c} a\right)-J_{\eta}\left(k_{c} a\right) Y_{\eta}^{\prime}\left(k_{c} b\right)}{J_{\eta}\left(k_{c} b\right) Y_{\eta}\left(k_{c} a\right)-J_{\eta}\left(k_{c} a\right) Y_{\eta}\left(k_{c} b\right)} \sin \left[\frac{m \pi(\phi+2 r)}{2(\pi-r-s-t)}\right]=0
\end{gathered}
$$

$$
\begin{aligned}
\tilde{X}_{1}^{I c}(n) & =\frac{1}{\pi\left(1+\delta_{n 0}\right)} \int_{-2(\pi-s-t)}^{2 s} X_{1}(\phi) \cos (n \phi) d \phi \\
\tilde{X}_{1}^{I s}(n) & =\frac{1}{\pi} \int_{-2(\pi-s-t)}^{2 s} X_{1}(\phi) \sin (n \phi) d \phi \\
\tilde{X}_{1}^{I I s}(m) & =\frac{1}{(\pi-t)} \int_{-2(\pi-s-t)}^{2 s} X_{1}(\phi) \sin \left[\frac{m \pi(\phi-2 s)}{2(\pi-t)}\right] d \phi \\
\tilde{X}_{2}^{I I s}(m) & =\frac{1}{(\pi-t)} \int_{0}^{2 s} X_{2}(\phi) \sin \left[\frac{m \pi(\phi-2 s)}{2(\pi-t)}\right] d \phi \\
\tilde{X}_{3}^{I I s}(m) & =\frac{1}{(\pi-t)} \int_{-2(\pi-s-t)}^{-2 r} X_{3}(\phi) \sin \left[\frac{m \pi(\phi-2 s)}{2(\pi-t)}\right] d \phi \\
\tilde{X}_{2}^{I I I s} & =\frac{1}{s} \int_{0}^{2 s} X_{2}(\phi) \sin \left[\frac{m \pi \phi}{2 s}\right] d \phi \\
\tilde{X}_{3}^{I V s}(m) & =\frac{1}{(\pi-r-s-t)} \int_{-2(\pi-s-t)}^{-2 r} X_{3}(\phi) \sin \left[\frac{m \pi(\phi+2 r)}{2(\pi-r-s-t)}\right] d \phi
\end{aligned}
$$




$$
\begin{aligned}
R_{k}(\phi) & =\frac{\sin \left[\frac{k \pi \phi}{2 s}\right]}{[\phi(4 s-\phi)]^{1 / 3}}, \quad k=1,2, \cdots \\
S_{k}(\phi) & =\frac{\sin \left[\frac{k \pi(\phi+2 r)}{2(\pi-r-s-t)}\right]}{[-(\phi+2 r)(\phi+4 \pi-4 s-4 t-2 r)]^{1 / 3}},
\end{aligned}
$$

The spectra of these basis functions in each of the subregions can be expressed in terms of Bessel functions of the first kind of order 1/6. They are given in Appendix A.

\section{Cutoff Wavenumbers of TE Modes}

The cutoff wavenumbers of the TE modes are determined following similar steps to those of Section III-A for the TM modes. The transverse components of the electromagnetic field of a TE mode are derivable from the axial component $H_{z}$. In each of the four subregions, $H_{z}$ is expanded in modal series which satisfy as much of the boundary conditions as possible. However, these expansions are not required to satisfy any specific term-wise boundary conditions at the interfaces. We, therefore, start from expansions (38a)-(38d), shown at the bottom of the page. Here, $l, \mu$, and $\eta$ are given by the same expressions as those of the TM case with the additional term $m=0$.

At the cutoff of a TE mode, the only nonvanishing components of the electromagnetic field which are tangential to the interfaces are $H_{z}$ and $E_{\phi}$, which is proportional to $\partial H_{z} / \partial \rho$.

The boundary conditions of the problem can be written in the following form:

$$
\begin{aligned}
E_{\phi}^{I}(\rho=d, \phi) & =0, \quad 2 s \leq \phi \leq 2(s+t) \\
E_{\phi}^{I I}(\rho=b, \phi) & =0, \quad 2(\pi-r-s-t) \leq \phi \leq 2 \pi \\
E_{\phi}^{I}(\rho=d, \phi) & =E_{\phi}^{I I}(\rho=b, \phi), \\
& \quad-2(\pi-s-t) \leq \phi \leq 2 s \\
E_{\phi}^{I I}(\rho=b, \phi) & =E_{\phi}^{I I I}(\rho=b, \phi), \quad 0 \leq \phi \leq 2 s \\
E_{\phi}^{I I}(\rho=b, \phi) & =E_{\phi}^{I V}(\rho=b, \phi), \\
\quad-2(\pi-s-t) \leq \phi \leq-2 r & \\
H_{z}^{I}(\rho=d, \phi) & =H_{z}^{I I}(\rho=b, \phi), \\
& -2(\pi-s-t) \leq \phi \leq 2 s \\
H_{z}^{I I}(\rho=b, \phi) & =H_{z}^{I I I}(\rho=b, \phi), \quad 0 \leq \phi \leq 2 s
\end{aligned}
$$

and

$$
\begin{aligned}
H_{z}^{I I}(\rho=b, \phi)=H_{z}^{I V} & (\rho=b, \phi), \\
& -2(\pi-s-t) \leq \phi \leq-2 r .
\end{aligned}
$$

Instead of following the MMT and deriving a matrix equation in the modal expansion coefficients of (38), we introduce unknown functions which describe the tangential electric field $E_{\phi}$ at the gaps of the interfaces. Let $Y_{1}(\phi), Y_{2}(\phi)$, and $Y_{3}(\phi)$ denote the true distributions of $E_{\phi}$ at the interfaces I-II, II-III, and II-IV, respectively. The function $Y_{2}(\phi)$ is nonzero only in the interval $0 \leq \phi \leq 2 s$ whereas $Y_{3}(\phi)$ is nonzero only when $2(s+t) \leq \phi \leq 2(\pi-r)$. To ensure that $E_{\phi}$ vanishes on the metallic portion of interface I-II, we also require that

$$
Y_{1}(\phi)=0, \quad 2 s \leq \phi \leq 2(s+t) .
$$

The boundary conditions (39) are now rewritten in the following form:

$$
\begin{aligned}
E_{\phi}^{I}(\rho=d, \phi) & =Y_{1}(\phi) \\
E_{\phi}^{I I}(\rho=d, \phi) & =Y_{1}(\phi)
\end{aligned}
$$

and

$$
\begin{aligned}
E_{\phi}^{I I}(\rho=b, \phi) & =Y_{2}(\phi) \\
E_{\phi}^{I I}(\rho=b, \phi) & =Y_{2}(\phi) \\
E_{\phi}^{I I}(\rho=b, \phi) & =Y_{3}(\phi) \\
E_{\phi}^{I V}(\rho=b, \phi) & =Y_{3}(\phi) .
\end{aligned}
$$

It is important to keep in mind that (41) holds only over the intervals of $\phi$ where the functions $Y_{i}$ are nonzero.

Using the modal expansions of $E_{\phi}$, which are obtained from (38), we can express the modal expansions in terms of the spectra of the functions $Y_{i}$ in the four regions. These expressions are then substituted in the continuity conditions of $H_{z}$ to derive the following three coupled integral equations in the functions $Y_{i}(\phi)$, shown in (42)-(44), at the bottom of the following page. Equations (42)-(44) hold over the same ranges of $\phi$ as (29)-(31), respectively. The transformed functions $\tilde{Y}$ are defined by (45a)-(45g), shown at the bottom of the following page. The functions $Y_{i}$ are expanded in series of basis functions which include the edge conditions at the four wedges of the two ridges. Let $P_{i}, Q_{i}$, and $R_{i}$ denote elements of these sets of basis functions such that

$$
Y_{1}(\phi)=\sum_{k=1}^{M} c_{k} P_{k}(\phi)
$$

$$
\begin{aligned}
H_{z}^{I}(\rho, \phi) & =\sum_{n=0}^{\infty} J_{n}\left(k_{c} \rho\right)\left[A_{n} \cos (n \phi)+B_{n} \sin (n \phi)\right] \\
H_{z}^{I I}(\rho, \phi) & =\sum_{m=0}^{\infty}\left[C_{m} J_{l}\left(k_{c} \rho\right)+D_{m} Y_{l}\left(k_{c} \rho\right)\right] \cos \left[\frac{m \pi(\phi-2 s)}{2(\pi-t)}\right] \\
H_{z}^{I I I}(\rho, \phi) & =\sum_{m=0}^{\infty} E_{m}\left[Y_{\mu}^{\prime}\left(k_{c} a\right) J_{\mu}\left(k_{c} \rho\right)-Y_{\mu}\left(k_{c} \rho\right) J_{\mu}^{\prime}\left(k_{c} a\right)\right] \cos \left[\frac{m \pi \phi}{2 s}\right] \\
H_{z}^{I V}(\rho, \phi) & =\sum_{m=0}^{\infty} F_{m}\left[Y_{\eta}^{\prime}\left(k_{c} a\right) J_{\eta}\left(k_{c} \rho\right)-Y_{\eta}\left(k_{c} \rho\right) J_{\eta}^{\prime}\left(k_{c} a\right)\right] \cos \left[\frac{m \pi(\phi+2 r)}{2(\pi-r-s-t)}\right]
\end{aligned}
$$




$$
\begin{aligned}
& Y_{2}(\phi)=\sum_{k=1}^{M} d_{k} Q_{k}(\phi) \\
& Y_{3}(\phi)=\sum_{k=1}^{M} e_{k} R_{k}(\phi) .
\end{aligned}
$$

Applying Galerkin's method to the integral equations (42)-(44) with (46), we obtain a set of linear homogeneous equations in the expansion coefficients $c_{i}, d_{i}$, and $e_{i}$ as follows:

$$
\begin{aligned}
{[A][c]+[B][d]+[C][e] } & =0 \\
{[D][c]+[E][d]+[F][e] } & =0 \\
{[G][c]+[H][d]+[I][e] } & =0 .
\end{aligned}
$$

The entries of the matrices appearing in this equation are given in Appendix B.

\section{Basis Functions for TE Modes}

The basis functions should include whatever information we have about the behavior of the functions they are used to represent, especially the nonanalytic or singular properties. In this case, $E_{\phi}$ is singular as $r^{-1 / 3}$ as the radial distance to the axis of the metallic wedge $r$ approaches zero [8]. Taking into account the presence of the two wedges, we propose the following set:

$P_{k}(\phi)=\frac{\cos \left[\frac{(k-1) \pi(\phi-2 s)}{2(\pi-t)}\right]}{\left[(2 s-\phi)(\phi+2(\pi-s-t)]^{1 / 3}\right.}, \quad k=1,2, \cdots$.

The inclusion of the proper edge conditions in the basis functions in Regions III and IV are achieved through a mirroring process. Indeed, by forcing the basis functions to be even with respect to the metallic surfaces located at $\phi=2 \mathrm{~s}$ and $\phi=2 s+2 t$, we are guaranteed that the edge conditions

$$
\begin{aligned}
& \sum_{m=0}^{\infty}\left\{\frac{\left[\tilde{Y}_{2}^{I I c}(m)+\tilde{Y}_{3}^{I I c}(m)\right]\left[Y_{l}^{\prime}\left(k_{c} d\right) J_{l}\left(k_{c} d\right)-J_{l}^{\prime}\left(k_{c} d\right) Y_{l}\left(k_{c} d\right)\right]}{J_{l}^{\prime}\left(k_{c} d\right) Y_{l}^{\prime}\left(k_{c} b\right)-J_{l}^{\prime}\left(k_{c} b\right) Y_{l}^{\prime}\left(k_{c} d\right)}+\frac{\tilde{Y}_{1}^{I I c}(m)\left[J_{l}^{\prime}\left(k_{c} d\right) Y_{l}\left(k_{c} b\right)-Y_{l}^{\prime}\left(k_{c} d\right) J_{l}\left(k_{c} b\right)\right]}{J_{l}^{\prime}\left(k_{c} d\right) Y_{l}^{\prime}\left(k_{c} b\right)-J_{l}^{\prime}\left(k_{c} b\right) Y_{l}^{\prime}\left(k_{c} d\right)}\right\} \\
& \cdot \cos \left[\frac{m \pi(\phi-2 s)}{2(\pi-t)}\right]-\sum_{n=0}^{\infty} \frac{J_{n}\left(k_{c} d\right)}{J_{n}^{\prime}\left(k_{c} d\right)}\left[\tilde{Y}_{1}^{I c}(n) \cos (n \phi)+\tilde{Y}_{1}^{I s}(n) \sin (n \phi)\right]=0 \\
& \sum_{m=0}^{\infty}\left\{\left[\tilde{Y}_{2}^{I I c}(m)+\tilde{Y}_{3}^{I I c}(m)\right] \frac{\left.Y_{l}^{\prime}\left(k_{c} b\right) J_{l}\left(k_{c} d\right)-J_{l}^{\prime}\left(k_{c} b\right) Y_{l}\left(k_{c} d\right)\right]}{J_{l}^{\prime}\left(k_{c} d\right) Y_{l}^{\prime}\left(k_{c} b\right)-J_{l}^{\prime}\left(k_{c} b\right) Y_{l}^{\prime}\left(k_{c} d\right)}+\tilde{Y}_{1}^{I I c}(m) \frac{\left.J_{l}^{\prime}\left(k_{c} b\right) Y_{l}\left(k_{c} b\right)-Y_{l}^{\prime}\left(k_{c} b\right) J_{l}\left(k_{c} b\right)\right]}{J_{l}^{\prime}\left(k_{c} d\right) Y_{l}^{\prime}\left(k_{c} b\right)-J_{l}^{\prime}\left(k_{c} b\right) Y_{l}^{\prime}\left(k_{c} d\right)}\right\} \\
& \cdot \cos \left[\frac{m \pi(\phi-2 s)}{2(\pi-t)}\right]-\sum_{m=0}^{\infty} \tilde{Y}_{2}^{I I I c}(m) \frac{J_{\mu}^{\prime}\left(k_{c} b\right) Y_{\mu}\left(k_{c} a\right)-J_{\mu}\left(k_{c} a\right) Y_{\mu}^{\prime}\left(k_{c} b\right)}{J_{\mu}^{\prime}\left(k_{c} b\right) Y_{\mu}^{\prime}\left(k_{c} a\right)-J_{\mu}^{\prime}\left(k_{c} a\right) Y_{\mu}^{\prime}\left(k_{c} b\right)} \cos \left[\frac{m \pi \phi}{2 s}\right]=0 \\
& \sum_{m=0}^{\infty}\left\{\left[\tilde{Y}_{2}^{I I c}(m)+\tilde{Y}_{3}^{I I c}(m)\right] \frac{\left.Y_{l}^{\prime}\left(k_{c} b\right) J_{l}\left(k_{c} d\right)-J_{l}^{\prime}\left(k_{c} b\right) Y_{l}\left(k_{c} d\right)\right]}{J_{l}^{\prime}\left(k_{c} d\right) Y_{l}^{\prime}\left(k_{c} b\right)-J_{l}^{\prime}\left(k_{c} b\right) Y_{l}^{\prime}\left(k_{c} d\right)}+\tilde{Y}_{1}^{I I c}(m) \frac{\left.J_{l}^{\prime}\left(k_{c} b\right) Y_{l}\left(k_{c} b\right)-Y_{l}^{\prime}\left(k_{c} b\right) J_{l}\left(k_{c} b\right)\right]}{J_{l}^{\prime}\left(k_{c} d\right) Y_{l}^{\prime}\left(k_{c} b\right)-J_{l}^{\prime}\left(k_{c} b\right) Y_{l}^{\prime}\left(k_{c} d\right)}\right\} \\
& \cdot \cos \left[\frac{m \pi(\phi-2 s)}{2(\pi-t)}\right]-\sum_{m=0}^{\infty} \tilde{Y}_{3}^{I V c}(m) \frac{J_{\eta}^{\prime}\left(k_{c} b\right) Y_{\eta}\left(k_{c} a\right)-J_{\eta}\left(k_{c} a\right) Y_{\eta}^{\prime}\left(k_{c} b\right)}{J_{\eta}^{\prime}\left(k_{c} b\right) Y_{\eta}^{\prime}\left(k_{c} a\right)-J_{\eta}^{\prime}\left(k_{c} a\right) Y_{\eta}^{\prime}\left(k_{c} b\right)} \cos \left[\frac{m \pi(\phi+2 r)}{2(\pi-r-s-t)}\right]=0
\end{aligned}
$$

$$
\begin{aligned}
\tilde{Y}_{1}^{I c}(n) & =\frac{1}{\pi\left(1+\delta_{n 0}\right)} \int_{-2(\pi-s-t)}^{2 s} Y_{1}(\phi) \cos (n \phi) d \phi \\
\tilde{Y}_{1}^{I s}(n) & =\frac{1}{\pi} \int_{-2(\pi-s-t)}^{2 s} Y_{1}(\phi) \sin (n \phi) d \phi \\
\tilde{Y}_{1}^{I I c}(m) & =\frac{1}{\left(1+\delta_{m 0}\right)(\pi-t)} \int_{-2(\pi-s-t)}^{2 s} Y_{1}(\phi) \cos \left[\frac{m \pi(\phi-2 s)}{2(\pi-t)}\right] d \phi \\
\tilde{Y}_{2}^{I I c}(m) & =\frac{1}{\left(1+\delta_{m 0}\right)(\pi-t)} \int_{0}^{2 s} Y_{2}(\phi) \cos \left[\frac{m \pi(\phi-2 s)}{2(\pi-t)}\right] d \phi \\
\tilde{Y}_{3}^{I I c}(m) & =\frac{1}{\left(1+\delta_{m 0}\right)(\pi-t)} \int_{-2(\pi-s-t)}^{-2 r} Y_{3}(\phi) \cos \left[\frac{m \pi(\phi-2 s)}{2(\pi-t)}\right] d \phi \\
\tilde{Y}_{2}^{I I I c} & =\frac{1}{\left(1+\delta_{m 0}\right) s} \int_{0}^{2 s} Y_{2}(\phi) \cos \left[\frac{m \pi \phi}{2 s}\right] d \phi \\
\tilde{Y}_{3}^{I V c}(m) & =\frac{1}{\left(1+\delta_{m 0}\right)(\pi-r-s-t)} \int_{-2(\pi-s-t)}^{-2 r} Y_{3}(\phi) \cos \left[\frac{m \pi(\phi+2 r)}{2(\pi-r-s-t)}\right] d \phi
\end{aligned}
$$




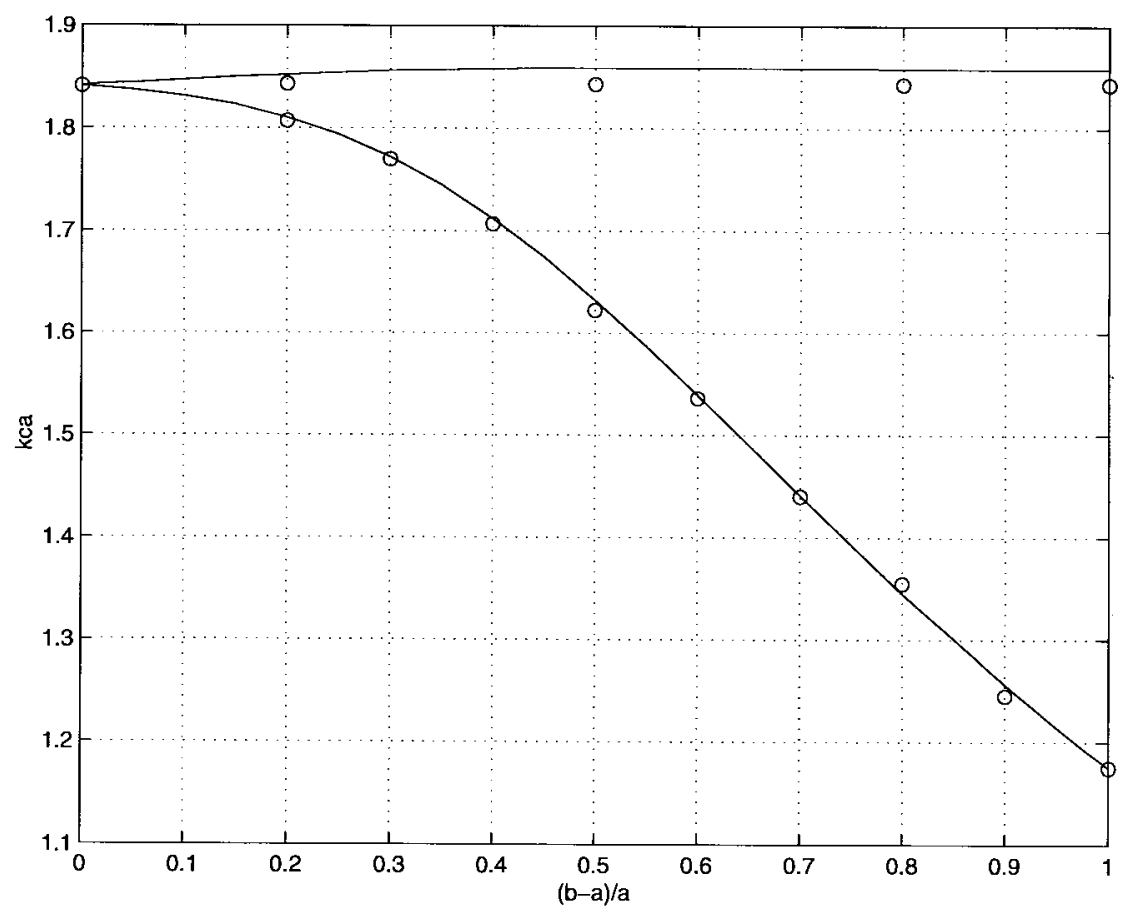

Fig. 3. Cutoff wavenumbers of the first TE modes of a single-ridge circular waveguide. The circles are from [1].

at these surfaces are accounted for. The edge conditions at the metallic wedges at $\phi=-2 r$ and $\phi=0$ are straightforwardly included through the weighting function. We finally have the following basis functions:

$$
\begin{aligned}
& Q_{k}(\phi)=\frac{\cos \left[\frac{(k-1) \pi \phi}{2 s}\right]}{[\phi(4 s-\phi)]^{1 / 3}}, \quad k=1,2, \cdots \\
& R_{k}(\phi)=\frac{\cos \left[\frac{(k-1) \pi(\phi+2 r)}{2(\pi-r-s-t)}\right]}{[-(\phi+2 r)(\phi+4 \pi-4 s-4 t-2 r)]^{1 / 3}}, \\
& k=1,2, \cdots .
\end{aligned}
$$

Taking into account the ranges of these basis functions, it can be easily verified that they include the proper edge conditions at all the metallic walls and edges. The spectra of these basis functions are given in Appendix B.

\section{Numerical Results AND Discussion}

The present technique is first applied to the case of a single-ridge circular waveguide.

To validate the theory and the computer code, the cutoff wavenumbers of the first two modes which correspond to the unperturbed fundamental mode of an empty circular waveguide $T E_{11}$ were computed using the present technique and are shown in Fig. 3. The circles are from [1] for the same dimensions of the structure. Good agreement between the two results is observed. Two basis functions were used in generating the data. The inner products which appear in the matrix elements in (22) reach convergence with 30 terms in the sums.

Cutoff wavelengths of higher order modes were also investigated for a number of dimensions of the ridge. Table I(a) and
TABLE I

Cutoff Wavelength $\lambda_{c} / 2 a$ of the First Few Modes of a Single Ridge for $b / a=0.9$ AND $\theta=0.02 \mathrm{rad}$ AS OBtaINED FROM the CIET AND THE MMT

\begin{tabular}{c|c|c}
\hline \hline Perturbed Mode & $\lambda_{c} / 2 a$ MMT $[3]$ & $\lambda_{c} / 2 a$ CIET \\
\hline TE11 & 1.7202 & 1.7093 \\
TE31 & 1.0446 & 1.0377 \\
TE51 & 0.7560 & 0.7560 \\
TE12 & 0.5896 & 0.5871 \\
TE32 & 0.4688 & 0.4747 \\
TM01 & 1.2887 & 1.2901 \\
\hline \hline
\end{tabular}

(a)

\begin{tabular}{c|c|c}
\hline \hline Perturbed Mode & $\lambda_{c} / 2 a M M T ~[3]$ & $\lambda_{c} / 2 a$ CIET \\
\hline TE11 & 1.9430 & 1.9226 \\
TE31 & 1.2019 & 1.2000 \\
TE51 & 0.8484 & 0.8509 \\
TE12 & 0.5943 & 0.5916 \\
TE32 & 0.4777 & 0.4761 \\
TM01 & 1.1943 & 1.2056 \\
\hline
\end{tabular}

(b)

(b) summarizes the results for cutoff wavelengths of the first few higher order modes along with the results obtained from the MMT [3]. Good agreement is again observed between the two sets of results.

The convergence of the numerical solution as the number of basis functions is increased is shown in Table II(a) and (b). It can be clearly seen that once enough basis functions are used for a cutoff to be encountered, the numerical solution converges rapidly, thereby confirming the judicious choice of the basis functions. The reason for the absence of some roots when the number of basis functions is not large enough, is due to the angular distribution of the fields of the modes and the nature of the chosen basis functions which are perturbed versions, through the edge conditions, of the modal fields 
TABLE II

Convergence of Cutoffs $\left(k_{c} a\right)$ OF THE First Few TM Modes of a Single-Ridge Versus the Number of BAsis Functions. $b / a=0.5, \theta=2.5^{\circ}$

\begin{tabular}{c|c|c|c}
\hline $\mathrm{M}=2$ & 3 & 4 & 5 \\
\hline 2.6215 & 2.5599 & 2.5599 & 2.5599 \\
3.8478 & 3.8478 & 3.8379 & 3.8379 \\
5.4890 & 4.1547 & 4.1547 & 4.1547 \\
& 5.6010 & 5.1756 & 5.1756 \\
& & 5.716 & 5.6010 \\
\hline
\end{tabular}

(a)

\begin{tabular}{c|c|c|c|c}
\hline \hline $\mathrm{M}=2$ & 3 & 4 & 6 & 8 \\
\hline 1.5743 & 1.5743 & 1.6342 & 1.6341 & 1.6341 \\
1.8389 & 1.8389 & 1.8389 & 1.8774 & 1.8807 \\
2.6711 & 2.6711 & 2.5805 & 2.6107 & 2.6118 \\
3.7371 & 3.7371 & 3.7371 & 3.0605 & 3.0846 \\
& & & 3.6898 & 3.7052 \\
\hline \hline
\end{tabular}

(b)

TABLE III

Cutoff Wavenumbers $\left(k_{c} a\right)$ of the First Eight TM Modes of AN Asymmetric Double-RIdge Structure FOR DifFerent VALUES OF $b / a$ AND $d / a \cdot r=t=2.5^{\circ}$ AND $2 s=130^{\circ}$

\begin{tabular}{c|r|r|r|r}
\hline \hline $\mathrm{b} / \mathrm{a}=0.95$ & 0.8 & 0.6 & 0.5 & 0.3 \\
\hline $\mathrm{d} / \mathrm{a}=0.90$ & 0.7 & 0.5 & 0.3 & 0.1 \\
\hline 2.402 & 2.420 & 2.570 & 2.796 & 3.316 \\
3.776 & 3.724 & 3.986 & 2.796 & 3.968 \\
3.876 & 4.072 & 4.654 & 4.200 & 4.614 \\
5.110 & 5.168 & 4.764 & 4.236 & 5.678 \\
5.126 & 5.254 & 5.638 & 5.168 & 5.940 \\
5.534 & 5.868 & 5.842 & 5.624 & 5.948 \\
6.338 & 6.548 & 5.962 & 5.824 & 6.706 \\
6.406 & 6.690 & 6.772 & 6.404 & 7.422 \\
\hline \hline
\end{tabular}

(a)

\begin{tabular}{c|r|r|r|r}
\hline \hline $\mathrm{b} / \mathrm{a}=0.95$ & 0.8 & 0.6 & 0.5 & 0.3 \\
\hline $\mathrm{d} / \mathrm{a}=0.90$ & 0.7 & 0.5 & 0.3 & 0.1 \\
\hline 1.830 & 1.756 & 1.570 & 1.362 & 1.088 \\
1.848 & 1.850 & 1.814 & 1.768 & 1.672 \\
3.032 & 2.866 & 2.594 & 2.446 & 2.340 \\
3.052 & 2.974 & 2.764 & 2.690 & 2.630 \\
3.832 & 3.818 & 3.630 & 3.598 & 3.582 \\
4.118 & 3.840 & 3.814 & 3.812 & 3.820 \\
4.166 & 4.126 & 3.982 & 3.958 & 3.942 \\
4.318 & 4.588 & 4.514 & 4.512 & 4.508 \\
\hline \hline
\end{tabular}

(b)

of the empty waveguide. It was noticed that a mode is encountered once a basis function with the proper angular distribution is used. For example, one or two basis functions are sufficient to determine the cutoff wavenumbers of the perturbed modes corresponding to the roots of $J_{0}\left(k_{c} a\right)$ or $J_{0}^{\prime}\left(k_{c} a\right)$. In addition, it is important to note that we analyzed both symmetries in one step which effectively reduces the number of contributing basis functions to a mode of a given symmetry. Had we taken advantage of the symmetry of the structure, the convergence of the solution would have been much faster. However, the fact that the technique recognizes the presence of the symmetry in the structure provides an additional confirmation of its validity.

The second structure investigated using the CIET consists of two asymmetric ridges in a circular waveguide (see Fig. 2).
TABLE IV

Convergence of Cutoffs of TM Modes With $k_{c} a \leq 6$ Versus the Number OF BASIS FUnCTIONS. $b / a=0.5, d / a=0.5, r=t=2.5^{\circ}$ AND $2 s=130^{\circ}$

\begin{tabular}{l|r|r|r|r|r|r}
\hline $\mathrm{M}=2$ & 3 & 4 & 5 & 6 & 7 & 8 \\
\hline 2.596 & 2.584 & 2.574 & 2.574 & 2.574 & 2.572 & 2.572 \\
4.598 & 4.056 & 3.990 & 3.990 & 3.988 & 3.986 & 3.986 \\
4.798 & 4.776 & 4.672 & 4.664 & 4.662 & 4.658 & 4.654 \\
5.738 & 5.052 & 4.776 & 4.768 & 4.766 & 4.764 & 4.764 \\
& 5.892 & 5.716 & 5.686 & 5.648 & 5.642 & 5.638 \\
& & 5.858 & 5.856 & 5.848 & 5.846 & 5.842 \\
& & & 5.992 & 5.976 & 5.964 & 5.963 \\
\hline
\end{tabular}

(a)

\begin{tabular}{l|r|r|r|r|r}
\hline \hline$\overline{\mathrm{M}=3}$ & 4 & 5 & 6 & 7 & 8 \\
\hline 1.498 & 1.528 & 1.562 & 1.562 & 1.566 & 1.570 \\
1.756 & 1.764 & 1.812 & 1.812 & 1.814 & 1.814 \\
2.096 & 2.364 & 2.564 & 2.568 & 2.584 & 2.594 \\
2.388 & 2.630 & 2.704 & 2.724 & 2.758 & 2.764 \\
3.078 & 3.328 & 3.368 & 3.584 & 3.602 & 3.630 \\
3.668 & 3.696 & 3.762 & 3.786 & 3.812 & 3.814 \\
3.726 & 3.832 & 3.834 & 3.892 & 3.966 & 3.982 \\
4.094 & 4.098 & 4.330 & 4.344 & 4.432 & 4.514 \\
\hline \hline
\end{tabular}

(b)

Tables III(a) and (b) summarize the results obtained using this present technique for two different sets of dimensions of the two ridges. For small ridges, our results reduce to those of the corresponding empty circular waveguide. These results were obtained using eight basis functions at each interface and 30 terms in the inner products.

The convergence of the numerical solution of the cutoff wavenumbers of the first few TM modes (i.e., those whose cutoff wavenumbers are such that $\left.k_{c} a \leq 6\right)$ is shown in Table IV(a). It is evident that even for this asymmetric structure, the CIET converges rapidly once enough basis functions for a root to be encountered are used. The convergence of the TE modes with $k_{c} a \leq 5$ is shown in Table IV(b).

We also report that spurious roots were encountered in the numerical solution for the TE modes in both the single- and double-ridge structures. These roots were always eliminated when a large enough number of basis functions were used. For example, if the presence of a root is suspicious, adding one or two more basis functions leads to its removal if it is indeed a spurious root.

\section{CONCLUSIONS}

The CIET was successfully applied to determine the cutoff wavenumbers of single- and double-ridge circular waveguide. The inclusion of the edge conditions in the basis functions at each of the sharp metallic wedges of the ridges makes the approach numerically efficient, as shown by the convergence study. The approach allows the inclusion of the singular behavior of the electromagnetic field at more than one ridge from the outset. The modes of the subregions are also all included in the theory by testing the sums in the inner products for convergence. The technique handles both symmetries in one step and is easily applicable to situations where more than two ridges are present. 


$$
\begin{aligned}
& \tilde{Q}_{k}^{I c}(n)=-\frac{G}{\left(1+\delta_{n 0}\right)}\left(1-\frac{t}{\pi}\right)\left\{\frac{J_{1 / 6}[|n(\pi-t)-(k \pi) / 2|]}{|(n(\pi-t)-(k \pi) / 2) / 2|^{1 / 6}} \sin \left[n(2 s-\pi+t)+\frac{k \pi}{2}\right]\right. \\
& \left.-\frac{J_{1 / 6}[|n(\pi-t)+(k \pi) / 2|]}{|[n(\pi-t)+(k \pi) / 2] / 2|^{1 / 6}} \sin \left[n(2 s-\pi+t)-\frac{k \pi}{2}\right]\right\} \\
& \tilde{Q}_{k}^{I s}(n)=G\left(1-\frac{t}{\pi}\right)\left\{\frac{J_{1 / 6}[|n(\pi-t)-(k \pi) / 2|]}{|[n(\pi-t)-(k \pi) / 2] / 2|^{1 / 6}} \cos \left[n(2 s-\pi+t)+\frac{k \pi}{2}\right]\right. \\
& \left.-\frac{J_{1 / 6}[|n(\pi-t)+(k \pi) / 2|]}{|[n(\pi-t)+(k \pi) / 2] / 2|^{1 / 6}} \cos \left[n(2 s-\pi+t)-\frac{k \pi}{2}\right]\right\} \\
& \tilde{Q}_{k}^{I I s}(m)=G\left\{\frac{J_{1 / 6}\left[|m-k| \frac{\pi}{2}\right]}{\left|\frac{m-k}{4} \pi\right|^{1 / 6}} \cos \left[\frac{m-k}{2} \pi\right]-\frac{J_{1 / 6}\left[(m+k) \frac{\pi}{2}\right]}{\left|\frac{m+k}{4} \pi\right|^{1 / 6}} \cos \left[\frac{m+k}{2} \pi\right]\right\} \\
& \tilde{R}_{k}^{I I s}(m)=G \frac{s}{\pi-t} \cos (k \pi)\left\{\frac{J_{1 / 6}\left[\left|\frac{m \pi s}{\pi-t}-k \pi\right|\right]}{\left|\frac{m \pi s}{2(\pi-t)}-\frac{k \pi}{2}\right|^{1 / 6}}-\frac{J_{1 / 6}\left[\left|\frac{m \pi s}{\pi-t}+k \pi\right|\right]}{\left|\frac{m \pi s}{2(\pi-t)}+\frac{k \pi}{2}\right|^{1 / 6}}\right\} \\
& \tilde{R}_{k}^{I I I s}(m)=G \cos [(m-k) \pi]\left\{\frac{J_{1 / 6}[|m-k| \pi]}{\left|\frac{m-k}{2} \pi\right|^{1 / 6}}-\frac{J_{1 / 6}[(m+k) \pi]}{\left|\frac{m+k}{2} \pi\right|^{1 / 6}}\right\} \\
& \tilde{S}_{k}^{I I s}(m)=G\left(1-\frac{s+r}{\pi-t}\right) \cos [(m-k) \pi]\left\{\frac{J_{1 / 6}\left[\left|\frac{m \pi(\pi-r-s-t)}{\pi-t}-k \pi\right|\right]}{\left|\frac{m \pi(\pi-r-s-t)}{2(\pi-t)}-\frac{k \pi}{2}\right|^{1 / 6}}-\frac{J_{1 / 6}\left[\left|\frac{m \pi(\pi-r-s+t)}{\pi-t}+k \pi\right|\right]}{\left|\frac{m \pi(\pi-r-s-t)}{2(\pi-t)}+\frac{k \pi}{2}\right|^{1 / 6}}\right\} \\
& \tilde{S}_{k}^{I V s}(m)=\tilde{R}_{k}^{I I I s}(m)
\end{aligned}
$$

$$
\begin{aligned}
{[A]_{i j}=} & \sum_{n=0}^{\infty} \frac{J_{n}^{\prime}\left(k_{c} d\right)}{J_{n}\left(k_{c} d\right)}\left[\tilde{Q}_{i}^{I c}(n) \tilde{Q}_{j}^{I c}(n)\left(1+\delta_{n 0}\right)+\tilde{Q}_{i}^{I s}(n) \tilde{Q}_{j}^{I s}(n)\right] \\
& -\left(1-\frac{t}{\pi}\right) \sum_{m=1}^{\infty} \frac{J_{l}^{\prime}\left(k_{c} d\right) Y_{l}\left(k_{c} b\right)-J_{l}\left(k_{c} b\right) Y_{l}^{\prime}\left(k_{c} d\right)}{J_{l}\left(k_{c} d\right) Y_{l}\left(k_{c} b\right)-J_{l}\left(k_{c} b\right) Y_{l}\left(k_{c} d\right)} \tilde{Q}_{i}^{I I s}(m) \tilde{Q}_{j}^{I I s}(m) \\
{[B]_{i j}=} & -\left(1-\frac{t}{\pi}\right) \sum_{m=1}^{\infty} \frac{J_{l}\left(k_{c} d\right) Y_{l}^{\prime}\left(k_{c} d\right)-Y_{l}\left(k_{c} d\right) J_{l}^{\prime}\left(k_{c} d\right)}{J_{l}\left(k_{c} d\right) Y_{l}\left(k_{c} b\right)-J_{l}\left(k_{c} b\right) Y_{l}\left(k_{c} d\right)} \tilde{Q}_{i}^{I I s}(m) \tilde{R}_{j}^{I I s}(m) \\
{[C]_{i j}=} & -\left(1-\frac{t}{\pi}\right) \sum_{m=1}^{\infty} \frac{J_{l}\left(k_{c} d\right) Y_{l}^{\prime}\left(k_{c} d\right)-Y_{l}\left(k_{c} d\right) J_{l}^{\prime}\left(k_{c} d\right)}{J_{l}\left(k_{c} d\right) Y_{l}\left(k_{c} b\right)-J_{l}\left(k_{c} b\right) Y_{l}\left(k_{c} d\right)} \tilde{Q}_{i}^{I I s}(m) \tilde{S}_{j}^{I I s}(m) \\
{[D]_{i j}=} & \left(1-\frac{t}{\pi}\right) \sum_{m=1}^{\infty} \frac{J_{l}^{\prime}\left(k_{c} b\right) Y_{l}\left(k_{c} b\right)-J_{l}\left(k_{c} b\right) Y_{l}^{\prime}\left(k_{c} b\right)}{J_{l}\left(k_{c} d\right) Y_{l}\left(k_{c} b\right)-J_{l}\left(k_{c} b\right) Y_{l}\left(k_{c} d\right)} \tilde{R}_{i}^{I I s}(m) \tilde{Q}_{j}^{I I s}(m) \\
{[E]_{i j}=} & \left(1-\frac{t}{\pi}\right) \sum_{m=1}^{\infty} \frac{J_{l}\left(k_{c} d\right) Y_{l}^{\prime}\left(k_{c} b\right)-J_{l}^{\prime}\left(k_{c} b\right) Y_{l}\left(k_{c} d\right)}{J_{l}\left(k_{c} d\right) Y_{l}\left(k_{c} b\right)-J_{l}\left(k_{c} b\right) Y_{l}\left(k_{c} d\right)} \tilde{R}_{i}^{I I s}(m) \tilde{R}_{j}^{I I s}(m) \\
& -\frac{s}{\pi} \sum_{m=1}^{\infty} \frac{J_{\mu}^{\prime}\left(k_{c} b\right) Y_{\mu}\left(k_{c} a\right)-J_{\mu}\left(k_{c} a\right) Y_{\mu}^{\prime}\left(k_{c} b\right)}{J_{\mu}\left(k_{c} b\right) Y_{\mu}\left(k_{c} a\right)-J_{\mu}\left(k_{c} a\right) Y_{\mu}\left(k_{c} b\right)} \tilde{R}_{i}^{I I s}(m) \tilde{R}_{j}^{I I I s}(m) \\
{[F]_{i j}=} & \left(1-\frac{t}{\pi}\right) \sum_{m=1}^{\infty} \frac{J_{l}\left(k_{c} d\right) Y_{l}^{\prime}\left(k_{c} b\right)-Y_{l}\left(k_{c} d\right) J_{l}^{\prime}\left(k_{c} b\right)}{J_{l}\left(k_{c} d\right) Y_{l}\left(k_{c} b\right)-J_{l}\left(k_{c} b\right) Y_{l}\left(k_{c} d\right)} \tilde{R}_{i}^{I s}(m) \tilde{S}_{j}^{I I s}(m)
\end{aligned}
$$




$$
\begin{aligned}
{[G]_{i j}=} & \left(1-\frac{t}{\pi}\right) \sum_{m=1}^{\infty} \frac{J_{l}^{\prime}\left(k_{c} b\right) Y_{l}\left(k_{c} b\right)-J_{l}\left(k_{c} b\right) Y_{l}^{\prime}\left(k_{c} b\right)}{J_{l}\left(k_{c} d\right) Y_{l}\left(k_{c} b\right)-J_{l}\left(k_{c} b\right) Y_{l}\left(k_{c} d\right)} \tilde{S}_{i}^{I I s}(m) \tilde{Q}_{j}^{I I s}(m) \\
{[H]_{i j}=} & \left(1-\frac{t}{\pi}\right) \sum_{m=1}^{\infty} \frac{J_{l}\left(k_{c} d\right) Y_{l}^{\prime}\left(k_{c} b\right)-Y_{l}\left(k_{c} d\right) J_{l}^{\prime}\left(k_{c} b\right)}{J_{l}\left(k_{c} d\right) Y_{l}\left(k_{c} b\right)-J_{l}\left(k_{c} b\right) Y_{l}\left(k_{c} d\right)} \tilde{S}_{i}^{I I s}(m) \tilde{R}_{j}^{I I s}(m) \\
{[I]_{i j}=} & \left(1-\frac{t}{\pi}\right) \sum_{m=1}^{\infty} \frac{J_{l}\left(k_{c} d\right) Y_{l}^{\prime}\left(k_{c} b\right)-J_{l}^{\prime}\left(k_{c} b\right) Y_{l}\left(k_{c} d\right)}{J_{l}\left(k_{c} d\right) Y_{l}\left(k_{c} b\right)-J_{l}\left(k_{c} b\right) Y_{l}\left(k_{c} d\right)} \tilde{S}_{i}^{I I s}(m) \tilde{S}_{j}^{I I s}(m) \\
& -\frac{\pi-r-s-t}{\pi} \sum_{m=1}^{\infty} \frac{J_{\eta}^{\prime}\left(k_{c} b\right) Y_{\eta}\left(k_{c} a\right)-J_{\eta}\left(k_{c} a\right) Y_{\eta}^{\prime}\left(k_{c} b\right)}{J_{\eta}\left(k_{c} b\right) Y_{\eta}\left(k_{c} a\right)-J_{\eta}\left(k_{c} a\right) Y_{\eta}\left(k_{c} b\right)} \tilde{S}_{i}^{I V s}(m) \tilde{S}_{j}^{I V s}(m)
\end{aligned}
$$

$$
\begin{aligned}
& \tilde{Q}_{k}^{I c}(n)=\frac{G\left(1-\frac{t}{\pi}\right)}{\left(1+\delta_{n 0}\right)}\left\{\frac{J_{1 / 6}\left[\left|n(\pi-t)-\frac{(k-1) \pi}{2}\right|\right]}{\left|n \frac{\pi-t}{2}-\frac{(k-1) \pi}{4}\right|^{1 / 6}} \cos \left[n(2 s-\pi+t)+\frac{(k-1) \pi}{2}\right]\right. \\
& \left.+\frac{J_{1 / 6}\left[\left|n(\pi-t)+\frac{(k-1) \pi}{2}\right|\right]}{\left|n \frac{\pi-t}{2}+\frac{(k-1) \pi}{4}\right|^{1 / 6}} \cos \left[n(2 s-\pi+t)-\frac{(k-1) \pi}{2}\right]\right\} \\
& \tilde{Q}_{k}^{I s}(n)=G\left(1-\frac{t}{\pi}\right)\left\{\frac{J_{1 / 6}\left[\left|n(\pi-t)-\frac{(k-1) \pi}{2}\right|\right]}{\left|n \frac{\pi-t}{2}-\frac{(k-1) \pi}{4}\right|^{1 / 6}} \sin \left[n(2 s-\pi+t)+\frac{(k-1) \pi}{2}\right]\right. \\
& \left.+\frac{J_{1 / 6}\left[\left|n(\pi-t)+\frac{(k-1) \pi}{2}\right|\right]}{\left|n \frac{\pi-t}{2}+\frac{(k-1) \pi}{4}\right|^{1 / 6}} \sin \left[n(2 s-\pi+t)-\frac{(k-1) \pi}{2}\right]\right\} \\
& \tilde{Q}_{k}^{I I c}(m)=\frac{G}{\left(1+\delta_{m 0}\right)}\left\{\frac{J_{1 / 6}\left[|m-k+1| \frac{\pi}{2}\right]}{\left|(m-k+1) \frac{\pi}{4}\right|^{1 / 6}} \cos \left[(m-k+1) \frac{\pi}{2}\right]+\frac{J_{1 / 6}\left[|m+k-1| \frac{\pi}{2}\right]}{\left|(m+k-1) \frac{\pi}{4}\right|^{1 / 6}} \cos \left[(m+k-1) \frac{\pi}{2}\right]\right\} \\
& \tilde{R}_{k}^{I I c}(m)=\frac{G}{1+\delta_{m 0}} \frac{s}{\pi-t} \cos [(k-1) \pi]\left\{\frac{J_{1 / 6}\left[\left|\frac{m \pi s}{\pi-t}-(k-1) \pi\right|\right]}{\left|\frac{m \pi s}{2(\pi-t)}-\frac{(k-1) \pi}{2}\right|^{1 / 6}}+\frac{J_{1 / 6}\left[\left|\frac{m \pi s}{\pi-t}+(k-1) \pi\right|\right]}{\left|\frac{m \pi s}{2(\pi-t)}+\frac{(k-1) \pi}{2}\right|^{1 / 6}}\right\} \\
& \tilde{R}_{k}^{I I I c}(m)=\frac{G}{\left(1+\delta_{m 0}\right)} \cos [(m-k+1) \pi]\left\{\frac{J_{1 / 6}[|m-k+1| \pi]}{\left|(m-k+1) \frac{\pi}{2}\right|^{1 / 6}}+\frac{J_{1 / 6}[|m+k-1| \pi]}{\left|(m+k-1) \frac{\pi}{2}\right|^{1 / 6}}\right\} \\
& \tilde{S}_{k}^{I I c}(m)=\frac{G}{1+\delta_{m 0}}\left(1-\frac{s+r}{\pi-t}\right) \cos [(m-k+1) \pi]\left\{\frac{J_{1 / 6}\left[\left|\frac{m \pi(\pi-r-s-t)}{\pi-t}-(k-1) \pi\right|\right]}{\left|\frac{m \pi(\pi-r-s-t)}{2(\pi-t)}-\frac{(k-1) \pi}{2}\right|^{1 / 6}}\right. \\
& \left.+\frac{J_{1 / 6}\left[\left|\frac{m \pi(\pi-r-s-t)}{\pi-t}+(k-1) \pi\right|\right]}{\left|\frac{m \pi(\pi-r-s-t)}{2(\pi-t)}+\frac{(k-1) \pi}{2}\right|^{1 / 6}}\right\}
\end{aligned}
$$

$\tilde{S}_{k}^{I V c}(m)=\tilde{R}_{k}^{I I I c}(m)$ 


$$
\begin{aligned}
& {[A]_{i j}=\sum_{n=0}^{\infty} \frac{J_{n}\left(k_{c} d\right)}{J_{n}^{\prime}\left(k_{c} d\right)}\left[\tilde{Q}_{i}^{I c}(n) \tilde{Q}_{j}^{I c}(n)\left(1+\delta_{n 0}\right)+\tilde{Q}_{i}^{I s}(n) \tilde{Q}_{j}^{I s}(n)\right]} \\
& -\left(1-\frac{t}{\pi}\right) \sum_{m=0}^{\infty} \frac{J_{l}\left(k_{c} d\right) Y_{l}^{\prime}\left(k_{c} b\right)-J_{l}^{\prime}\left(k_{c} b\right) Y_{l}\left(k_{c} d\right)}{J_{l}^{\prime}\left(k_{c} d\right) Y_{l}^{\prime}\left(k_{c} b\right)-J_{l}^{\prime}\left(k_{c} b\right) Y_{l}^{\prime}\left(k_{c} d\right)} \tilde{Q}_{i}^{I I c}(m) \tilde{Q}_{j}^{I I c}(m)\left(1+\delta_{m 0}\right) \\
& {[B]_{i j}=-\left(1-\frac{t}{\pi}\right) \sum_{m=0}^{\infty} \frac{J_{l}^{\prime}\left(k_{c} d\right) Y_{l}\left(k_{c} d\right)-J_{l}\left(k_{c} d\right) Y_{l}^{\prime}\left(k_{c} d\right)}{J_{l}^{\prime}\left(k_{c} d\right) Y_{l}^{\prime}\left(k_{c} b\right)-J_{l}^{\prime}\left(k_{c} b\right) Y_{l}^{\prime}\left(k_{c} d\right)} \tilde{Q}_{i}^{I I c}(m) \tilde{R}_{j}^{I I c}(m)\left(1+\delta_{m 0}\right)} \\
& {[C]_{i j}=-\left(1-\frac{t}{\pi}\right) \sum_{m=0}^{\infty} \frac{J_{l}^{\prime}\left(k_{c} d\right) Y_{l}\left(k_{c} d\right)-J_{l}\left(k_{c} d\right) Y_{l}^{\prime}\left(k_{c} d\right)}{J_{l}^{\prime}\left(k_{c} d\right) Y_{l}^{\prime}\left(k_{c} b\right)-J_{l}^{\prime}\left(k_{c} b\right) Y_{l}^{\prime}\left(k_{c} d\right)} \tilde{Q}_{i}^{I I c}(m) \tilde{S}_{j}^{I I c}(m)\left(1+\delta_{m 0}\right)} \\
& {[D]_{i j}=\left(1-\frac{t}{\pi}\right) \sum_{m=0}^{\infty} \frac{J_{l}\left(k_{c} b\right) Y_{l}^{\prime}\left(k_{c} b\right)-J_{l}^{\prime}\left(k_{c} b\right) Y_{l}\left(k_{c} b\right)}{J_{l}^{\prime}\left(k_{c} d\right) Y_{l}^{\prime}\left(k_{c} b\right)-J_{l}^{\prime}\left(k_{c} b\right) Y_{l}^{\prime}\left(k_{c} d\right)} \tilde{R}_{i}^{I I c}(m) \tilde{Q}_{j}^{I I c}(m)\left(1+\delta_{m 0}\right)} \\
& {[E]_{i j}=\left(1-\frac{t}{\pi}\right) \sum_{m=0}^{\infty} \frac{J_{l}^{\prime}\left(k_{c} d\right) Y_{l}\left(k_{c} b\right)-J_{l}\left(k_{c} b\right) Y_{l}^{\prime}\left(k_{c} d\right)}{J_{l}^{\prime}\left(k_{c} d\right) Y_{l}^{\prime}\left(k_{c} b\right)-J_{l}^{\prime}\left(k_{c} b\right) Y_{l}^{\prime}\left(k_{c} d\right)} \tilde{R}_{i}^{I I c}(m) \tilde{R}_{j}^{I I c}(m)\left(1+\delta_{m 0}\right)} \\
& -\frac{s}{\pi} \sum_{m=0}^{\infty} \frac{J_{\mu}\left(k_{c} b\right) Y_{\mu}^{\prime}\left(k_{c} a\right)-J_{\mu}^{\prime}\left(k_{c} a\right) Y_{\mu}\left(k_{c} b\right)}{J_{\mu}^{\prime}\left(k_{c} b\right) Y_{\mu}^{\prime}\left(k_{c} a\right)-J_{\mu}^{\prime}\left(k_{c} a\right) Y_{\mu}^{\prime}\left(k_{c} b\right)} \tilde{R}_{i}^{I I I c}(m) \tilde{R}_{j}^{I I I c}(m)\left(1+\delta_{m 0}\right) \\
& {[F]_{i j}=\left(1-\frac{t}{\pi}\right) \sum_{m=0}^{\infty} \frac{J_{l}^{\prime}\left(k_{c} d\right) Y_{l}\left(k_{c} b\right)-J_{l}\left(k_{c} b\right) Y_{l}^{\prime}\left(k_{c} d\right)}{J_{l}^{\prime}\left(k_{c} d\right) Y_{l}^{\prime}\left(k_{c} b\right)-J_{l}^{\prime}\left(k_{c} b\right) Y_{l}^{\prime}\left(k_{c} d\right)} \tilde{R}_{i}^{I I c}(m) \tilde{S}_{j}^{I I c}(m)\left(1+\delta_{m 0}\right)} \\
& {[G]_{i j}=\left(1-\frac{t}{\pi}\right) \sum_{m=0}^{\infty} \frac{J_{l}\left(k_{c} b\right) Y_{l}^{\prime}\left(k_{c} b\right)-J_{l}^{\prime}\left(k_{c} b\right) Y_{l}\left(k_{c} b\right)}{J_{l}^{\prime}\left(k_{c} d\right) Y_{l}^{\prime}\left(k_{c} b\right)-J_{l}^{\prime}\left(k_{c} b\right) Y_{l}^{\prime}\left(k_{c} d\right)} \tilde{S}_{i}^{I I c}(m) \tilde{Q}_{j}^{I I c}(m)\left(1+\delta_{m 0}\right)} \\
& {[H]_{i j}=\left(1-\frac{t}{\pi}\right) \sum_{m=0}^{\infty} \frac{J_{l}^{\prime}\left(k_{c} d\right) Y_{l}\left(k_{c} b\right)-J_{l}\left(k_{c} b\right) Y_{l}^{\prime}\left(k_{c} d\right)}{J_{l}^{\prime}\left(k_{c} d\right) Y_{l}^{\prime}\left(k_{c} b\right)-J_{l}^{\prime}\left(k_{c} b\right) Y_{l}^{\prime}\left(k_{c} d\right)} \tilde{S}_{i}^{I I c}(m) \tilde{R}_{j}^{I I c}(m)\left(1+\delta_{m 0}\right)} \\
& {[I]_{i j}=\sum_{m=0}^{\infty}\left(1+\delta_{m 0}\right)\left\{\left(1-\frac{t}{\pi}\right) \frac{J_{l}^{\prime}\left(k_{c} d\right) Y_{l}\left(k_{c} b\right)-J_{l}\left(k_{c} b\right) Y_{l}^{\prime}\left(k_{c} d\right)}{J_{l}^{\prime}\left(k_{c} d\right) Y_{l}^{\prime}\left(k_{c} b\right)-J_{l}^{\prime}\left(k_{c} b\right) Y_{l}^{\prime}\left(k_{c} d\right)} \tilde{S}_{i}^{I I c}(m) \tilde{S}_{j}^{I I c}(m)\right.} \\
& \left.-\frac{(\pi-r-s-t)}{\pi} \frac{J_{\eta}\left(k_{c} b\right) Y_{\eta}^{\prime}\left(k_{c} a\right)-J_{\eta}^{\prime}\left(k_{c} a\right) Y_{\eta}\left(k_{c} b\right)}{J_{\eta}^{\prime}\left(k_{c} b\right) Y_{\eta}^{\prime}\left(k_{c} a\right)-J_{\eta}^{\prime}\left(k_{c} a\right) Y_{\eta}^{\prime}\left(k_{c} b\right)} \tilde{S}_{i}^{I V c}(m) \tilde{S}_{j}^{I V c}(m)\right\}
\end{aligned}
$$

\section{APPENDIX A}

In this appendix, we give the expressions of the transformed basis functions for TM modes as well as the entries of the matrices in (34). The transformations of the basis functions as defined by (32) are expressed in terms of Bessel functions of the first kind of order $1 / 6$ [9] [G=0.5 $\left.\Gamma\left(\frac{1}{2}\right) \Gamma\left(\frac{2}{3}\right)\right]$, shown in (A.1)-(A.7), at the top of page 490. The entries of the matrices in (34) follow from taking the dot products of the integral equations (29)-(31) against the basis functions (A.8)-(A.13), shown at the bottom of page 490, and (A.14)-(A.16), shown at the top of the previous page.

\section{APPENDIX B}

In this appendix, we give the expressions of the transformed basis functions for TE modes as well as the entries of the matrices in (47). The transformations of the basis functions as defined by (45) are expressed in terms of Bessel functions of the first kind of order $1 / 6[9]\left[G=0.5 \Gamma\left(\frac{1}{2}\right) \Gamma\left(\frac{2}{3}\right)\right]$, shown in (B.1)-(B.7), at the bottom of the previous page. The entries of the matrices in (47) follow from taking the dot products of the integral equations (42)-(44) against the basis functions (B.8)-(B.16), shown at the top of the page.

\section{ACKNOWLEDGMENT}

The authors would like to thank G. Steiner for helping with the figures.

\section{REFERENCES}

[1] R. Behe and P. Brachat, "Compact duplexer-polarizer with semicircular waveguide," IEEE Trans. Antenna Propagat., vol. 39, pp. 1222-1224, Aug. 1991.

[2] W. Sun and C. A. Balanis, "Analysis and design of quadruple ridged waveguides," IEEE Trans. Microwave Theory Tech., vol. 42, pp. 2201-2207, Dec. 1994.

[3] U. Balaji and R. Vahldieck, "Radial mode matching analysis of ridged circular waveguides," IEEE Trans. Microwave Theory Tech., vol. 44, pp. 1183-1186, July 1996.

[4] S. Xiao, R. Vahldieck, and M. Guglielmi, "Field theory analysis of circular ridge waveguides with partial dielectric filling," in 1995 IEEE MTT-S Int. Microwave Symp. Dig., vol. 1, Orlando, FL, May 1995, pp. 265-268.

[5] A. S. Omar, A. Jöstingmeier, C. Rieckmann, and S. Lütgert, “ Application of the GSD technique to the analysis of slot-coupled waveguides," IEEE Trans. Microwave Theory Tech., vol. 42, pp. 2139-2147, Nov. 1994. 
[6] R. F. Harrington, Field Computation by the Moment Methods. Orlando, FL: Krieger, 1987, p. 72.

[7] V. Labay and J. Bornemann, "Matrix singular value decomposition for pole free solutions of homogeneous matrix equations as applied to numerical modeling," IEEE Microwave Guided Wave Lett., vol. 2, pp. 49-51, Feb. 1992.

[8] R. E. Collin, Field Theory of Guided Waves. New York: IEEE Press, 1991.

[9] I. S. Gradshteyn and I. M. Ryznik, Tables of Integrals, Series, and Products, 5th ed. New York: Academic, 1994

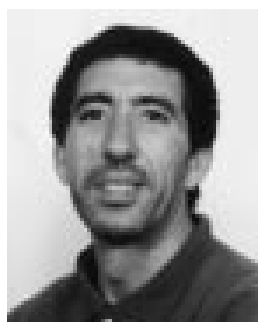

Smain Amari received the D.E.S. degree in physics and electronics from Constantine University, Constantine, Algeria, in 1985, and the M.S. degree in electrical engineering and the Ph.D. degree in physics from Washington University, St. Louis, MO, in 1989 and 1994, respectively.

Since 1994, he has been with the Department of Electrical and Computer Engineering, University of Victoria, Victoria, B.C., Canada. He is interested in numerical methods in electromagnetics, numerical analysis, applied mathematics, applied physics, and application of quantum field theory in quantum many-particle systems.
Rüdiger Vahldieck (M'85-SM'86) received the Dipl.-Ing. and the Dr.-Ing. degrees in electrical engineering from the University of Bremen, Bremen, Germany, in 1980 and 1983, respectively.

From 1984 to 1986, he was a Research Associate at the University of Ottawa, Ottawa, Ont., Canada. In 1986, he joined the University of Victoria, Victoria, B.C., Canada, where in 1991, he became a full Professor in the Department of Electrical and Computer Engineering. From 1992 to 1993 , he was a Visiting Scientist at the Ferdinand-Braun Institute für Hochfrequenztechnik, Berlin, Germany. Since October 1996, he has been the Chair for Field Theory at the Laboratory for Electromagnetic Fields and Microwave Electronics, Swiss Federal Institute of Technology, Zurich, Switzerland. He has published over 150 technical papers. His research interests include numerical methods to model electromagnetic fields for computer-aided design of microwave, millimeter wave, and opto-electronic integrated circuits. $\mathrm{He}$ is also interested in the design and simulation of devices and subsystems for broad-band fiber-optic communication systems.

Dr. Vahldieck is on the editorial board of the IEEE TRANSACTIONS ON MicROWAVE THEORY AND TeCHNIQUES, and since 1992, has served on the Technical Program Committee of the IEEE International Microwave Symposium. He was the co-recipient (along with three co-authors) of the Outstanding Publication Award of the Institution of Electronic and Radio Engineers in 1983.

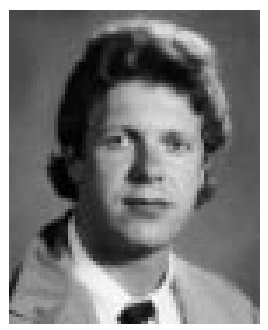

Jens Bornemann (M'87-SM'90) was born in Hamburg, Germany, on May 26, 1952. He received the Dipl.-Ing. and the Dr.-Ing. degrees, from the University of Bremen, Bremen, Germany, in 1980 and 1984, respectively, both in electrical engineering.

From 1980 to 1983, he was a Research and Teaching Assistant in the Microwave Department, University of Bremen, where he worked on quasi-planar waveguide configurations and computer-aided $E$ plane filter design. In 1985, after a two-year period as a Consulting Engineering, he joined the University of Bremen again, as an Assistant Professor. Since April 1988, he has been with the University of Victoria, Victoria, B.C., Canada, where he is currently a Professor in the Department of Electrical and Computer Engineering. His research activities include microwave/millimeter-wave components and systems design, and problems of electromagnetic-field theory in integrated circuits and radiating structures. He co-authored Waveguide Components for Antenna Feed Systems. Theory and Design (Norwood, MA: Artech House, 1993) and has authored/coauthored over 120 technical papers. He serves on the editorial board of the International Journal of Numerical Modeling.

Dr. Bornemann is a Registered Professional Engineer in the Province of British Columbia, Canada, and has been a Fellow of the British Columbia Advanced Systems Institute from 1992 to 1995. He serves on the editorial board of the IEEE TRAnsactions on Microwave Theory and TeChNiQues. He was one of the recipients of the 1983 A. F. Bulgin Premium of the Institution of Electronic and Radio Engineers.
Serine Catreux was born in Rennes, France, on November 8, 1973. received the Diploma of Engineering degree in electrical engineering from currently working toward the Ph.D. degree in electrical engineering at the University of Victoria, Victoria, B.C., Canada.

Her main research interests are statistical and adaptive signal processing and its applications in communication. 